\title{
ORBITAL OPTIMIZATION OF INTERPLANETARY TRAJECTORIES WITH ENVIRONMENTAL PERTURBATIONS
}

\author{
A Thesis \\ presented to \\ the Faculty of California Polytechnic State University, \\ San Luis Obispo
}

\author{
In Partial Fulfillment \\ of the Requirements for the Degree \\ Master of Science in Aerospace Engineering
}

by

Eric Woods

June 2018 
(C) 2018

Eric Woods

ALL RIGHTS RESERVED 


\section{COMMITTEE MEMBERSHIP}

TITLE:

Orbital Optimization of Interplanetary Trajectories with Environmental Perturbations

AUTHOR:

Eric Woods

DATE SUBMITTED: June 2018

COMMITTEE CHAIR: Kira Abercromby, Ph.D.

Associate Professor of Aerospace Engineering

COMMITTEE MEMBER: Colleen Kirk, Ph.D.

Professor of Mathematics

COMmitTeE MEMBER: Jordi Puig Suari, Ph.D.

Professor of Aerospace Engineering

COMMITTEE MEMBER: Eric Mehiel, Ph.D.

Professor of Aerospace Engineering 


\begin{abstract}
Orbital Optimization of Interplanetary Trajectories with Environmental Perturbations

Eric Woods
\end{abstract}

For a detailed analysis of orbital optimization, it is desired to incorporate a spacecraft environment model in order to have maximum confidence that the analysis will produce an accurate trajectory. Such a model requires the addition of orbital perturbations, or small forces acting on the spacecraft throughout its trajectory that can eventually accumulate in large distances over time. The optimization method that this thesis is concerned with is STOpS (Spacecraft Trajectory Optimization Suite), a Matlab optimizer created by Timothy J. Fitzgerald that utilizes an Island Model Paradigm with five different optimization algorithms. STOpS was originally built to model trajectories with the two body equations of motion. A Lambert's method was utilized to link the spacecraft trajectory from planet to planet, and a flyby section was created for the hyperbolic gravity assist trajectories. A cost function was then used to evaluate the best combination of $\Delta \mathrm{V}$, time of flight, synodcity, flyby altitude, and heliocentric energy. This work is primarily concerned with adding the dynamics created by perturbations into Lambert's problem as well as the gravity assist trajectories. The improved analysis creates a more robust solution for dealing with optimized interplanetary trajectories. Two proven trajectories will be focused on for the main analysis of this thesis which are the trajectories taken by Voyager 2 in the tour of the solar system as well as Cassini's mission to Saturn. When perturbations were added to the analysis of these missions, STOpS was able to find trajectories which met both $\Delta \mathrm{V}$ and time of flight requirements for each mission. For the optimization of each of these missions the key dates of departure, flyby, and arrival at all the planets varied by no more than one year from the true trajectories of Voyager 2 and Cassini. 


\section{ACKNOWLEDGMENTS}

Thanks to:

- I would first like to thank Dr. A for her continued support throughout my graduate studies as my thesis advisor. I have learned a great deal from the many insights that she has provided over the years as a professor at Cal Poly.

- I would also like to thank all of the faculty in aerospace engineering as I would not be where I am today without their continued knowledge and support.

- Finally, thank you to my family for supporting me throughout these long college years and having the trust in me that I could accomplish all my goals as an aspiring engineer. 


\section{TABLE OF CONTENTS}

Page

LIST OF TABLES . . . . . . . . . . . . . . . . . .

LIST OF FIGURES . . . . . . . . . . . . . . . . . . xi CHAPTER

1 Introduction . . . . . . . . . . . . . . . . . 1

1.1 Nomenclature . . . . . . . . . . . . . . . . . . 1

1.2 Statement of Problem . . . . . . . . . . . . . . . 2

1.3 Purpose of Study . . . . . . . . . . . . . . . . 3

1.4 Existing Optimization Programs . . . . . . . . . . . . . 4

2 Review of Orbital Mechanics . . . . . . . . . . . . . . . . 6

2.1 Elliptical Sections . . . . . . . . . . . . . . 6

2.2 Hyperbolic Sections . . . . . . . . . . . . . . . . . . 7

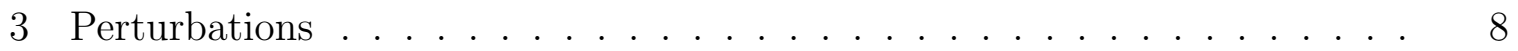

3.1 Perturbations in the Spacecraft Environment . . . . . . . . . . 8

3.1.1 N-body Gravitation . . . . . . . . . . . . . 8

3.1.2 Solar Radiation Pressure . . . . . . . . . . . . . . 9

3.1.3 Atmospheric Drag . . . . . . . . . . . . . . 10

$3.1 .4 \mathrm{~J}_{2} \ldots \ldots \ldots \ldots \ldots$

3.1.5 General Relativity ................ 12

3.2 Implementation . . . . . . . . . . . . . . . . . 13

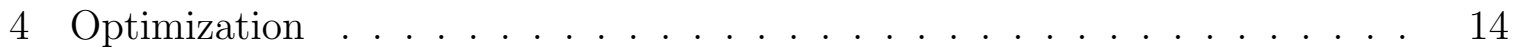

4.1 Methodology . . . . . . . . . . . . . . . 14

4.2 Island Topology . . . . . . . . . . . . . . . . . 15

4.3 Genetic Algorithms . . . . . . . . . . . . . . . . . 17

4.3.1 Population Size . . . . . . . . . . . . . . 18

4.3.2 Chance of Mutation . . . . . . . . . . . . . . 18

4.3 .3 Number of Generations . . . . . . . . . . . . . . . . . 19

4.4 Differential Evolution . . . . . . . . . . . . . . . . . . . . 19

4.5 Particle Swarm Optimization . . . . . . . . . . . . . 20 
4.5 .1 Particle Motion . . . . . . . . . . . . . . . . . . 20

4.5 .2 Informants . . . . . . . . . . . . . . . . . . . 21

4.6 Ant Colony Optimization . . . . . . . . . . . . . . . . . . . . . 21

5 Lambert's Problem . . . . . . . . . . . . . . . . . . . . . . 23

5.1 The Two Body Lambert's Problem . . . . . . . . . . . . . . 23

5.2 The Perturbed Lambert's Problem _... . . . . . . . . . . 26

5.2 .1 Shepperd's Method . . . . . . . . . . . . . . . 26

$5.2 .2 \quad$ Runge Kutta Fehlburg 78 . . . . . . . . . . . . . . . . 32

5.2.3 Determination of the True Trajectory . . . . . . . . . . . 37

5.2.4 Consideration for Lambert's Perturbations . . . . . . . . . . 38

6 Gravitational Assists . . . . . . . . . . . . . . . . . . . . . 40

6.1 The Two Body Gravity Assist . . . . . . . . . . . . . . . 40

6.2 The Perturbed Gravity Assist . . . . . . . . . . . . . . . . . 42

7 Results and Analysis . . . . . . . . . . . . . . . . . 47

7.1 Perturbation Magnitude Comparison _... . . . . . . . . 47

7.2 Analysis of Test Case Trajectory . . . . . . . . . . . . . . . 48

7.3 Cassini Trajectory . . . . . . . . . . . . . . . 50

7.3 .1 Cassini Background . . . . . . . . . . . . . . . . 50

7.3.2 Cassini Optimization Setup _. . . . . . . . . . . . 51

7.3.3 Cassini Analysis Without Perturbations . . . . . . . . . 54

7.3.4 Cassini Analysis With Perturbations . . . . . . . . . . 59

7.4 Voyager 2 Trajectory . . . . . . . . . . . . . . . . 62

7.4.1 Voyager 2 Background . . . . . . . . . . . . . . 62

7.4.2 Voyager 2 Optimization Setup . . . . . . . . . . . . . 63

7.4.3 Voyager 2 Analysis Without Perturbations . . . . . . . . . 65

7.4.4 Voyager 2 Analysis With Perturbations . . . . . . . . . 68

8 Conclusions . . . . . . . . . . . . . . . . . . . . . . . . . . 72

8.1 Future Work . . . . . . . . . . . . . . . . . . . . . 74

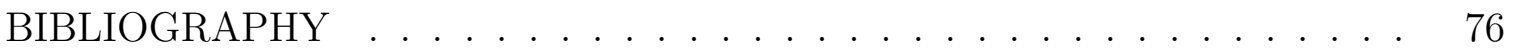
APPENDICES

A STOpS User Guide . . . . . . . . . . . . . . 78

A.1 Optimization Panels . . . . . . . . . . . . . . 78 
A.1.1 Island Topology . . . . . . . . . . . . . . . . . 78

A.1.2 Trajectory Information . . . . . . . . . . . . . . . 79

A.1.3 Optimization Options . . . . . . . . . . . . . . . . . . 79

A.1.4 Cost Function Options ～. . . . . . . . . . . . . . . . . . . 79

A.1.5 Actual Mission . . . . . . . . . . . . . . . . . 80

A.2 Results of Analysis . . . . . . . . . . . . . . . 80 


\section{LIST OF TABLES}

Table

7.1 Perturbation Magnitude Comparison at One Astronomical Unit . . 48

7.2 Flight Parameters of Trajectory From Earth to Saturn . . . . . . . 49

7.3 Cost Parameter Comparison for Cassini's Unperturbed Trajectory . 57

7.4 Flight Time Comparison for Cassini's Unperturbed Trajectory . . . 58

7.5 Cost Parameter Comparison for Cassini's Perturbed Trajectory . . 61

7.6 Flight Time Comparison for Cassini's Perturbed Trajectory . . . . 61

7.7 Cost Parameters for Voyager 2's Unperturbed Trajectory . . . . . . 67

7.8 Flight Times for Voyager 2's Unperturbed Trajectory . . . . . . . 68

7.9 Cost Parameter Comparison for Voyager 2's Perturbed Trajectory . 70

7.10 Flight Time Comparison for Voyager 2's Perturbed Trajectory . . . 70 


\section{LIST OF FIGURES}

Figure

$4.1 \quad$ Island Topology . . . . . . . . . . . . . . . . . . . . . 16

$6.1 \quad$ Hyperbolic Gravity Assist Maneuver . . . . . . . . . . . . . . 40

7.1 Trajectory From Earth to Saturn . . . . . . . . . . . . . . . . 49

7.2 Cassini's Trajectory Panel . . . . . . . . . . . . . . . . . 52

7.3 Cassini's Cost Function Panel . . . . . . . . . . . . . . . . . 53

7.4 Cassini's Optimization Panel . . . . . . . . . . . . . . . . 54

7.5 Cassini's Optimized Trajectory . . . . . . . . . . . . 56

$7.6 \quad$ Actual Cassini Trajectory . . . . . . . . . . . . . 56

7.7 Cassini's Optimized Trajectory With Perturbations . . . . . . . 60

7.8 Voyager 2 Trajectory Panel . . . . . . . . . . . . . . . 63

7.9 Voyager 2 Cost Function Panel . . . . . . . . . . . . . . . . 64

7.10 Voyager 2's Optimization Panel . . . . . . . . . . . . . . 65

7.11 Voyager 2's Optimized Trajectory . . . . . . . . . . . . 66

7.12 Actual Voyager 2 Trajectory . . . . . . . . . . . . . . 66

7.13 Voyager 2's Optimized Trajectory With Perturbations _... . . . 69 
Chapter 1

INTRODUCTION

\section{$1.1 \quad$ Nomenclature}

$\Delta \mathrm{V}$ change in velocity magnitude by spacecraft

G gravitational constant

$\vec{r} \quad$ position vector

$\vec{v} \quad$ velocity vector

$\mu \quad$ standard gravitational parameter

$\mathrm{T}$

period of orbit

$\mathrm{a}$

semi-major axis of orbit

W

solar constant

c

speed of light

$\rho$

atmospheric density

$C_{D}$

coefficient of drag

$J_{2}$

zonal harmonic constant

$\epsilon$

orbital energy

$\Theta$

state transition matrix

$R_{S O I}$

sphere of influence

$\delta$

hyperbolic turn angle

$r_{p}$

radius of perigee

e

eccentricity 


\subsection{Statement of Problem}

With any orbital trajectory there are an infinite number of paths that a spacecraft can take to get from point A to point B. While these trajectories may all be mathematically feasible, they are not all equally useful for application in spacecraft missions. If it is decided that time of flight is an important parameter in a mission, then the trajectory that gets one spacecraft from Earth to Mars in 100 days is likely better than the one that takes 1 year to complete. This is where the method of optimization is particularly useful. Given any cost function, the user can decide which parameters are important to optimize and STOpS (Spacecraft Trajectory Optimization Suite) will search for a global minimum for this specified cost function. For this thesis, a test case trajectory will be studied from Earth to Saturn with a Jupiter gravity assist. The real spacecraft missions of Cassini as well as the trajectory taken by Voyager 2 on its tour of the solar system starting in August 20, 1977 will be also be analyzed in order to determine the accuracy of the optimization process developed in this work. In the test case trajectory, it is desired to find a feasible orbit that has been optimized to preserve as much fuel as possible while maintaining a relatively short time of flight. In the case of both Cassini and Voyager 2's mission, these trajectories have already been well defined and proven to be successful. If this optimization process is to be shown to be robust, then STOpS should be able to calculate a trajectory that arrives at the same planets that both Cassini and Voyager 2 did with a similar $\Delta \mathrm{V}$ and time of flight when compared to the actual spacecraft missions. The addition of perturbations should also produce a reasonable trajectory that agrees with the trajectory taken by Cassini and Voyager 2 . 


\subsection{Purpose of Study}

Because of the wide range of possible trajectories, it can be particularly difficult for mission designers to find the optimal solution for their requirements. There is the rather large problem that spacecraft have $\Delta \mathrm{V}$ restrictions and therefor can only carry a certain amount of fuel making many trajectories unworkable. Then there is also a heavy emphasis on time of flight because many missions, specifically interplanetary missions, place a large importance on mission lifetime. It is then important that most of that lifetime not be spent traveling to the desired destination. However it is not enough to simply find the shortest possible trajectory. Because of the ever changing position of the planets it may be desired to wait for a gravity assist or sacrifice some amount of time for better fuel efficiency. It may in fact be most optimal to wait years before launching in order to obtain the most desired trajectory. What all of these considerations result in is very few orbital trajectories that will even be possible in order to reach the desired destination. Then there is the next level of difficulty in finding the best trajectory among the small population of viable ones. Perhaps the largest obstacles in obtaining this optimal trajectory are the large number of variables that need to be considered as well as the large search space for trajectories. This leads to the fact that a detailed optimized interplanetary trajectory cannot be performed analytically but instead must be performed numerically. This is where STOpS gains most of its strength in that it can evaluate thousands of trajectories with minimal inputs from the user. From this method, orbital optimization is able to help mission designers ensure that their mission is both successful and able to garner the most scientific potential with the time that is allotted, by calculating an optimal trajectory tailored to their needs. 


\subsection{Existing Optimization Programs}

Trajectory design and optimization is a field of study that has gained a great deal of interest over the years due to its importance in the spacecraft industry. As a result, many orbital optimization programs have been developed over the years by a variety of different companies and universities. Most of these software systems are either proprietary are cost a large amount of money in order to access.

Some of the most prominent optimization systems developed over the years include VARITOP, CHEBYTOP, MIDAS, SEPSPOT, GESOP, ASTOS, and Copernicus. MIDAS for example uses a patched conic system where the spacecraft utilizes flybys around planets or other small celestial bodies. The user specifies the number of planetary flybys prior to launch [13]. MIDAS is very similar to STOpS in this way as STOpS also utilizes patch conics and requires the user to input the desired flyby planets prior to optimization.

VARITOP is an optimization system which utilizes a general two-body, suncentered trajectory design and optimization program. VARITOP focuses on trajectories which do not make use of or require instantaneous velocity changes. However it does not perform well with missions which require very low thrust on the spacecraft such as the use of ion propulsion [9].

Another of NASA's optimization options to be discussed is SEPSPOT. This program was designed to handle electric thrust which would produce slow changes in velocity over time. However, it is only capable of minimizing trip time. This work fo-

cuses on missions which are capable of producing higher thrust and so STOpS would not be able to handle these types of trajectories [9].

Copernicus is perhaps the most developed optimization software tool which started as a prototype in 2001. Copernicus was developed by the University of Texas at 
Austin. Further updates and improvements on this software have continued until current day. Copernicus is capable of solving a wide range of trajectory problems such as planet or moon centered trajectories, libration point trajectories, planetmoon transfers and tours, and all types of interplanetary and asteroid/comet missions. Copernicus is capable of incorporating multiple gravitational bodies into the optimization process as is required for its use of libration point trajectories. It is also capable of incorporating solar radiation pressure and drag into a spacecraft's trajectory [19]. Unfortunately Copernicus is only available free of charge through the affiliation of other NASA centers, government contractors, and universities, under the terms of a US government purpose license.

The current version of STOpS is unique to many of these optimization systems in that it incorporates environmental perturbations into the dynamics of the spacecrafts motion. Many of these older optimization systems specifically are only capable of solving for two body Sun centered trajectories. In addition, STOpS is able to handle many high thrust options which other optimization systems cannot utilize including multiple revolutions, and the option for prograde and retrograde trajectories which can be specified through Lambert's problem. With the inclusion of a graphical user interface it is also straight forward to obtain the desired trajectory with minimal effort from the user. While STOpS still does not have the capabilities of an optimization system such as Copernicus it has the benefit of not being proprietary and free to access. 
Chapter 2

\section{REVIEW OF ORBITAL MECHANICS}

In order to understand optimization of orbital trajectories it is first important to be well acquainted with orbital mechanics in general. This requires first understanding many of the driving principles in the two body problem. From this frame work it is then possible to build in the dynamics from orbital perturbations. These principles will later give greater understanding to Lambert's problem which is the method that will eventually be used to create the desired interplanetary trajectories. In the two body problem there are two main types of orbits which will constitute the needed interplanetary trajectory taken by the spacecraft. These two types of orbits being an elliptical trajectory from one planet to another and a hyperbolic trajectory for gravity assists around intermediate planets.

\subsection{Elliptical Sections}

For the STOpS method, elliptical trajectories are positioned so that the Sun lies at one of the focus points of the orbital ellipse. It is also important to note that the reference frame that is being used to measure the spacecraft from derives from the Sun during this time. While in a real spacecraft interplanetary trajectory there will be other forces present acting on the spacecraft, the dominant force during this elliptical trajectory is the gravitational force from the Sun. For the purpose of the STOpS optimization process it is also important to understand Lambert's method. It should be noted that orbital optimization can be used without the use of Lambert's method but it is particularly useful in this method as it can calculate a wide range

of interplanetary trajectories very quickly. Lambert's method is important for the 
elliptical orbits because it is used to create the path from one planet to another. It works by first knowing the desired initial and final positions, and a known time of flight. Through a geometric solution, the initial and final velocities can be found by first finding the Lagrange variables. Once the velocity vectors are obtained, the orbit can be propagated and the path of the spacecraft can be determined up until it reaches its next destination.

\subsection{Hyperbolic Sections}

Once the spacecraft becomes sufficiently close to a planet, the orbit is modeled as a hyperbola with the planet at the hyperbolas focus point. This process begins once the spacecraft reaches the sphere of influence of the planet, also noted as the point in space where the gravitational force from the planet becomes greater than the gravitational force due to the Sun [17]. The reference frame also changes at this point to be centered around the body frame of the planet. Meaning that all positions and velocities at this point in the trajectory are measured with respect to the planet and not the Sun. These gravity assists are important in the optimization process as they can give a large change in velocity without exerting any fuel from the spacecraft by using the gravity of the planet to change the flight path angle of the spacecraft. Hyperbolic orbits have a higher energy than elliptical orbits which means the gravitational force from the planet will not be strong enough to capture the spacecraft as it flies by. This results in intermediate planets acting as pit stops for the spacecraft rather than destination points where the spacecraft will continue on its trajectory to the next planet. 
Chapter 3

PERTURBATIONS

\subsection{Perturbations in the Spacecraft Environment}

Because the focus of this work is the addition of orbital perturbations into the STOpS optimization analysis, these environmental perturbations must first be defined. There are many different perturbations, or small forces present in the spacecraft environment. The two body problem is concerned only with the dynamics of the spacecraft under the presence of the Sun's gravitational force. The Sun is the dominant force acting on the spacecraft over its trajectory, so it is useful to simplify the model to only include this force. The analysis developed in this work however has been further improved to include dynamics produced from gravitational forces of other planets and moons, solar radiation pressure originating from the Sun, atmospheric drag, J2, and the effects of general relativity. These perturbations are added to the Sun's acceleration to describe a more complete picture of the spacecraft's dynamics in a real interplanetary mission. A brief overview and explanation of these forces is first needed before it is possible to implement them into the analysis.

\subsubsection{N-body Gravitation}

N-body forces are described as the gravitational forces from other celestial bodies [17]. These forces act in the same way as the dominant force from the Sun. They exert an acceleration obeying the same laws as the Sun described by Newton's law of universal gravitation discussed earlier. 


$$
\vec{a}_{b o d y}=-\mu \frac{\vec{r}_{m s}}{\left\|\vec{r}_{m s}\right\|^{3}}
$$

However because the Sun is the center of the reference frame for the elliptical trajectories and the planets are not, their equations take a slightly different form. Given that there are many minor gravitational bodies that can accelerate the spacecraft, these accelerations must be summed in order to obtain the net acceleration of the system.

$$
\begin{gathered}
\vec{r}_{m s}=\vec{r}_{M s}-\vec{r}_{M m} \\
\vec{a}_{b o d y}=\sum_{i=1}^{n}-\mu \frac{\vec{r}_{M s}-\vec{r}_{M m i}}{\left\|\vec{r}_{M s}-\vec{r}_{M m i}\right\|^{3}}
\end{gathered}
$$

Here the first subscript denotes the starting point of the position vector while the second denotes the end point of the position vector. $M$ represents the main gravitational body which in this case is the Sun, $\mathrm{m}$ represents the minor gravitational bodies which in this case are the planets, s represents the spacecraft, and n specifies the number of minor gravitational bodies contributing to the n-body perturbation.

\subsubsection{Solar Radiation Pressure}

The acceleration due to solar radiation pressure is produced by the electromagnetic force. A large number of photons originating from the Sun exert a force on the exposed area of the spacecraft over the course of its trajectory. This acceleration becomes larger the closer the spacecraft is to the Sun as the photons become more 
concentrated [17]. This perturbation results in a radial force outward that can be modeled by equations 3.4-3.5.

$$
\cos \beta=\frac{\vec{r}_{M s} \cdot \vec{n}}{\left\|\vec{r}_{M s}\right\|\|\vec{n}\|}
$$

where $\vec{r}_{M s}$ is the position vector from the Sun to the spacecraft and $\vec{n}$ is the vector normal to the spacecraft surface.

$$
\vec{a}_{S R P}=\frac{W A(1+\alpha) \vec{r}_{M s}}{c\left\|\vec{r}_{M s}\right\|^{3}} \cos \beta
$$

where $\mathrm{W}$ is the solar constant, $\mathrm{A}$ is the area of the spacecraft facing the Sun, $\alpha$ is the fraction of light reflected by the spacecraft, and c is the speed of light.

\subsubsection{Atmospheric Drag}

Drag is the force produced by the interaction between the spacecraft and a planet's atmosphere. Because the number of molecules in interplanetary space is sufficiently small, this force will only be modeled when the spacecraft comes within close proximity to a planet's atmosphere [17]. The acceleration due to drag can be modeled by equation 3.6.

$$
\vec{a}_{\text {drag }}=-\frac{1}{2} \rho C_{D} \frac{A}{m}\|\vec{v}\| \vec{v}
$$

where $\rho$ is the density of the fluid, $C_{D}$ is the coefficient of drag, $\vec{v}$ is the veloc- 
ity vector of the spacecraft relative to a rotating atmosphere, $\mathrm{A}$ is the area of the spacecraft facing the velocity vector, and $\mathrm{m}$ is the mass of the spacecraft.

\subsection{4 $\mathrm{J}_{2}$}

In mathematics it can be shown through Stokes' theorem that the gravitational acceleration due to a point mass is identical to the gravitational acceleration produced by a sphere of uniform density so long as the particle is outside the radius of the sphere [11]. However because planetary bodies are not perfectly spherical and are better modelled as oblate spheroids, there is a perturbed acceleration that must be calculated into the dynamics of the spacecraft. These perturbations can be referred to as spherical harmonics which then produce a series expansion model for the gravitational acceleration [17]. The first of these terms excluding the perfectly spherical acceleration is the $J_{2}$ term. This $J_{2}$ term dominates in magnitude over the other terms $\left(J_{3}, J_{4}\right.$, etc) and so only $J_{2}$ will be used to model the perturbations due the spherical harmonics of the gravitational body. The $J_{2}$ of the Sun will be included for the elliptical trajectories while the $J_{2}$ of the flyby planet will be included for

the hyperbolic trajectories. The acceleration produced by $J_{2}$ can be modeled by the equations of motion 3.7-3.10.

$$
\begin{aligned}
& \ddot{x}_{J 2}=\frac{-3 J_{2} \mu R^{2}}{2\|\vec{r}\|^{5}}\left(1-\frac{5 z^{2}}{\|\vec{r}\|^{2}}\right) x \\
& \ddot{y}_{J 2}=\frac{-3 J_{2} \mu R^{2}}{2\|\vec{r}\|^{5}}\left(1-\frac{5 z^{2}}{\|\vec{r}\|^{2}}\right) y
\end{aligned}
$$




$$
\begin{gathered}
\ddot{z}_{J 2}=\frac{-3 J_{2} \mu R^{2}}{2\|\vec{r}\|^{5}}\left(3-\frac{5 z^{2}}{\|\vec{r}\|^{2}}\right) z \\
\vec{a}_{J 2}=\left(\ddot{x}_{J 2}, \ddot{y}_{J 2}, \ddot{z}_{J 2}\right)
\end{gathered}
$$

where $J_{2}$ is a zonal harmonic constant defined by the gravitational body, $\mu$ is the standard gravitational parameter, and $\mathrm{R}$ is the average radius of the gravitational body.

\subsubsection{General Relativity}

General relativity is responsible for changing the dynamics of a two body system when the main gravitational body becomes sufficiently large. Although the Sun is not large enough to cause drastic changes in the classical equations of motion, the effect of this phenomenon is still noticeable and measurable [16]. A first order linear approximation of the perturbation due to general relativity is given below by equation 3.11 .

$$
a_{\vec{G} R}=-\frac{\mu}{\|\vec{r}\|^{3} c^{2}}\left(\left(\frac{4 \mu}{\|\vec{r}\|}-\|\vec{v}\|^{2}\right)+4(\vec{v} \cdot \vec{r}) \vec{v}\right)
$$

where $\mu$ is the standard gravitational parameter and $\mathrm{c}$ is the speed of light. 


\subsection{Implementation}

The key of this work is then adding these perturbations into the orbital mechanics techniques already present within the STOpS code. The particular challenge is with Lambert's method which is primarily an orbital mechanics solution to the two body problem. In order to include the dynamics from the orbital perturbations, an iterative process must be used in which an initial guess is given and continually refined after propagating the trajectory until its error is within an acceptable value of the true solution. This method will be looked into with more detail when examining Lambert's solution with perturbations.

Although this code is designed to be more robust specifically with respect to the spacecraft environment, certain assumptions must still be made when analyzing the perturbations. Because of the architecture of the initial STOpS code, it is necessary to continue to use patched conics for the optimization analysis. Patched conics are particularly useful when examining trajectories that move from planet to planet. However they simplify the dynamics of the system by changing the problem from a Sun focused reference frame where the Sun is the dominant gravitational body to a planet focused reference frame where the planet is the dominant gravitational body.

As a result the whole trajectory cannot be run as one long propagation but rather it must be split into sections and propagated for each leg from where the trajectory last left off. This however poses problems for where it is needed to turn the perturbations off when the spacecraft nears its next target planet. 


\section{Chapter 4}

\section{OPTIMIZATION}

\subsection{Methodology}

For many problems within engineering it is desirable to find the optimal solution. A method can be described as optimal if it obtains the most desirable set of outputs which are specified before the optimization process begins. In terms of analysis, optimality can be described as the maximum or minimum of a function over its domain. Of course for any given function there can be an infinite number of local maxima and minima scattered throughout the domain that are not as desirable as the truly optimized solution. It is then the goal of the optimization process to determine the absolute maxima and minima rather than a local set. This can pose a problem as it can often be difficult for optimization methods to branch out from a local minima due to the fact that optimization methods converge to solutions depending on the rate of change of the function. It is then important for these evolutionary algorithms to be able to branch out and search larger areas of the functions domain to ensure that they find the most optimized solution possible.

STOpS was developed with its optimization process utilizing a collection of evolutionary algorithms. It works by first obtaining a population of values that are chosen by an educated guess which are dependent upon the inputs specified by the user. In this case the population of values are flight times between planet to planet. Through multiple calculations it can then associate a cost to each of these population members. In the case of optimal orbital trajectories this cost is derived from $\Delta \mathrm{V}$ used by the spacecraft, transfer time, synodicity, radius of perigee for flyby maneuvers, and final heliocentric energy. Each of these parameters must then also be assigned a weight 
to determine which parameters, if any, are more important to optimize. The cost function for this orbital optimization problem is specified below by equation 4.1.

$$
J=W_{1} \Delta V+W_{2} t+W_{3} \Delta V_{\text {syn }}+W_{4} R_{p}+W_{5} \epsilon_{\text {end }}
$$

where $W_{1-5}$ are the weights associated with the cost function parameters, $\Delta \mathrm{V}$ is the total change in velocity performed solely by the spacecraft's fuel, $R_{p}$ is the radius of perigee of each flyby, $\Delta V_{\text {syn }}$ is the velocity synodicity, $\epsilon_{\text {end }}$ is the final heliocentric energy of the spacecraft at the end of its trajectory, and t is the total time of flight. It should also be noted that for specific missions, this cost function can be modified in terms of the weights given as well as which of these five parameters should be included. Each evolutionary algorithm method within STOpS has a way of selecting for the best cost and then branching out by throwing the worst solutions in the population out and replacing them with new ones to be evaluated. This process is called a change in the generation of the population and can occur many times as specified by the user. After all of the generations are completed a migration occurs that swaps the solutions to a new evolutionary algorithm method. Finally the best method can be selected for and all parameters can be specified and plotted. Also important to note is that within these evolutionary algorithms, there are many different parameters that the user can modify depending on the type of mission desired or depending on time constraints on code evaluation.

\subsection{Island Topology}

STOpS uses a generalized island model, which is a method used in optimization that allows multiple algorithms to run, then allows them to share and compare their solutions. They can then use this information to explore new areas of the search space 
or update their population to find a solution faster. This allows different algorithms to work together and feed off of each others strengths and overcome each others weaknesses [18]. Each method constitutes one island, and the layout of these islands is referred to as a topology. Each algorithm can share solutions with another member of the topology during a migration. The user can specify the number of migrations which will occur which can further improve upon the solution. Although more migrations will result in more sharing of results and thus a more optimized solution, it will also create a longer computational time for the optimization process. An example topology can be seen in figure 4.1 with each number within the topology representing a different evolutionary algorithm.

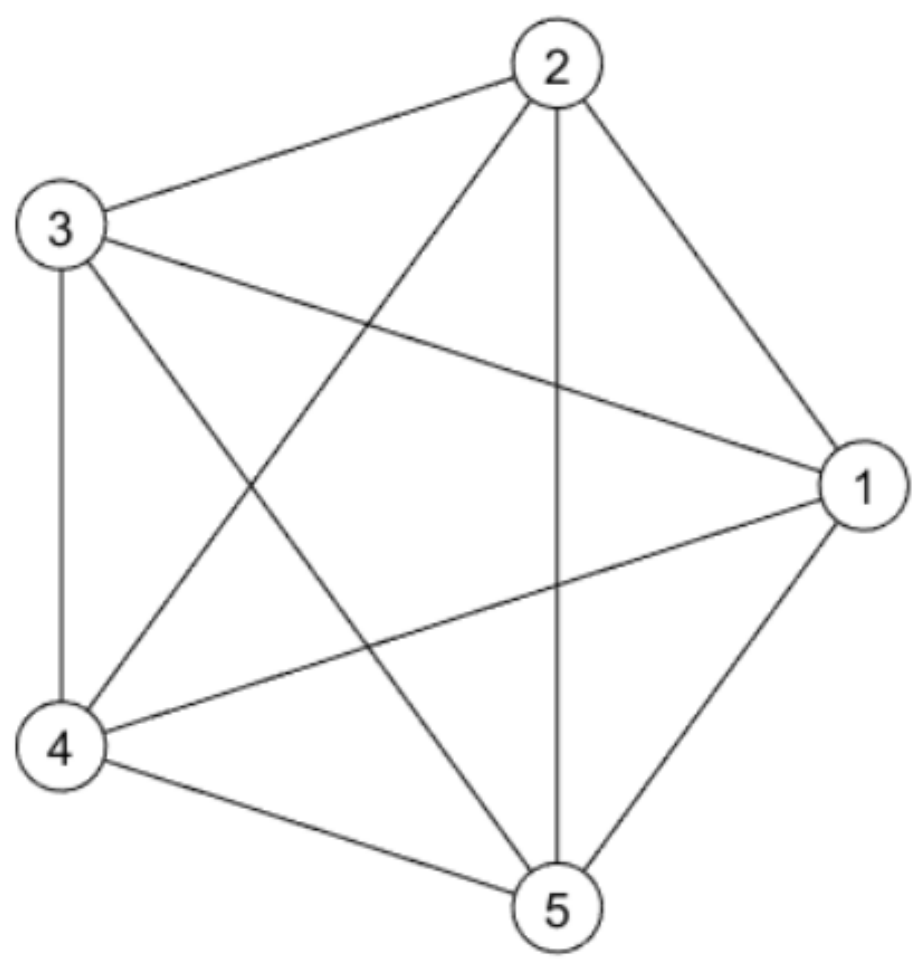

Figure 4.1: Island Topology

A brief overview of the evolutionary algorithms used in the STOpS method are 
included here. The reader is encouraged to review a more detailed methodology on the evolutionary algorithms as well as the optimization process used within STOpS in Timothy J. Fitzgerald's thesis [6].

\subsection{Genetic Algorithms}

The first of the optimization methods used in this work is the Genetic Algorithm also referred to as GA. This genetic algorithm is based on the works, Genetic Algorithms in Search, Optimization, and Machine Learning by David E. Goldberg [7], and Practical Genetic Algorithms by Randy L. Haupt and Sue Ellen Haupt [8]. The reader is encouraged to review the works of these authors for a full walk-through of the methodology within the genetic algorithm. What genetic algorithms seek to model is biological optimization similar to the theory of Darwin and survival of the fittest by natural selection. Genetic algorithms start with a span of random solutions which can be referred to as a population similar to an animal population. They then use some selection method to decide which solutions to use in mating to create a new group of solutions. This group of solutions would then go on to become offspring, or part of the next generation. This process continues until the best member of the current generation has a solution that meets some criteria, or the algorithm can run for a fixed number of generations. The search process for new solutions is random similar to the case of mutation in animal populations. However because only the best solutions survive every generation, the algorithm will often arrive at a highly optimized solution despite the randomness in the search process. A more detailed view of the parameters within the genetic algorithm can now be outlined. 


\subsubsection{Population Size}

Population size is an important aspect of the setup to optimize these trajectories. It has already been established that the populations are a set of trajectory times from one planet to another. Each member of the population will have transfer times corresponding to one more than the number of flybys specified by the user. For example if it was desired to find a trajectory from Earth to Saturn with one flyby around Jupiter, then every member of the population would have two trajectory times. Population size is important in evolutionary algorithms because it represents how many different trajectories are being evaluated. A larger population size means that it is more likely to find an optimized solution. However there is the draw back that a larger population leads to more time to computationally evaluate. For the purpose of this analysis the population was set to 200 members. This proved to be large enough so that the same optimized solution could be found reliably and small enough so that the computational time remained at a feasible value.

\subsubsection{Chance of Mutation}

Chance of mutation is another important aspect in evolutionary algorithms as it determines how the algorithm searches for better solutions. Every member of the population has a chance to mutate, which changes the values of the transfer times. Too low of a mutation rate will cause the search to become stagnant. The algorithm will not be able to find better solutions because it isn't given the chance to evaluate any new trajectories. On the other hand too large of a mutation rate can result in better solutions being thrown out of the analysis. This would lead to a kind of random evaluation process that would never converge on an optimized solution. The mutation rate base lined for STOpS was chosen to be 40 percent and has been shown to reliably produce the same optimized solution given a constant set of inputs. 


\subsubsection{Number of Generations}

Number of generations for the population is another important input for the user to specify in the optimization process. This will specify how many times the population will change. Like mutation rate, the number of generations helps to sort out the best solutions from the worst ones. With each successive generation, more of the low cost trajectories will be kept and the higher cost trajectories are thrown out. This process is similar to a kind of genetic drift seen in animal populations. In general a larger number of generations will lead to a more optimized solution as the algorithm has more steps to refine its solution. However too large of a generation number will result in a larger computational time needed to evaluate all the trajectories. In this work the number of generations was base lined at 30 as by this point the cost becomes stagnant for most trajectories and no further improvements can be found.

\subsection{Differential Evolution}

The next method in this work, known as Differential Evolution (DE) was adapted from the source: Differential Evolution: A Practical Approach to Global Optimization by Kenneth Price, Rainer M. Storm, and Jouni A. Lampinen [12]. This section provides a summary of their methodology and the reader is encouraged to reference these resources for a more detailed overview of the methodology. The process that DE follows is roughly similar to genetic algorithms in that they both start with an initial random population and have offspring that move on to the next generation. The difference between the methods is how they determine which members of the population move on to the next generation, and how they actually change those members. In the differential evolution algorithm, an entirely new population of mutant members is formed and then a specific percentage of them replace the old members of the population through what is referred to as crossover. This is not what would be referred 
to as a next generation but instead a blend of an existing population with a newly mutated population.

\subsection{Particle Swarm Optimization}

The next algorithm in the optimization process is known as Particle Swarm Optimization or PSO. The particular method used in this work was adapted from one source: Particle Swarm Optimization by Maurice Clerc [2]. The interested reader is encouraged to reference that book for more detailed explanations. This algorithm can be modelled by the behavior of bees in their search for pollen. The bees are forced to begin their search for pollen by flying randomly in different directions. Eventually some number of bees will find locations with pollen. These bees can then communicate these optimal areas to the rest of the bees when they come into contact with them. This process continues until all the bees know where the best area to find pollen is. The difference between the PSO algorithm and the bees is that the bees have to fly to and from one set location which would be their hive. The PSO algorithm instead gives each particle its own random initial position and a random initial velocity, and from there they explore the work space which is defined for them. The particles communicate with each other, and a few things influence a particles velocity: its own velocity, the best solution it has found, and the best solution that a different particle has told it about.

\subsubsection{Particle Motion}

The user can specify different aspects of the particle's motion in Optimization Options in the STOpS interface. There are three aspects that affect a particles movement: its own current velocity denoted by $\mathrm{v}$, its knowledge of the best solution that it has seen which is a location denoted by $\mathrm{p}$, and the knowledge of the best solution that 
an informant particle has seen which is a location denoted by g.

\subsubsection{Informants}

The user can set the number of informants $\mathrm{K}$ which are able to communicate with each other during their search. It is important to specify a reasonable value for this parameter because if all particles speak to each other on each iteration, then the current best overall solution found will dominate the choices made by all particles. This could potentially lead to premature convergence or a solution that is not truly optimal. On the other hand if not enough particles are able to communicate, then each particle could be left to explore on its own. This would eventually make the process turn into a purely random search which is not useful.

\subsection{Ant Colony Optimization}

The next algorithm explored in this work is known as Ant Colony Optimization. This method is modelled from the behavior of ants because they are very efficient at finding food sources and communicating the location to the rest of the colony. This optimization process is similar to the others in that it seeks to explore real world behavior to arrive at a solution. The methodology behind this algorithm is based off the work of Ant Colony Optimization by Marco Dorigo and Thomas Stutzle [4]. As is the case for the other algorithms, the reader is encouraged to review the material within this reference to obtain a better overview of the optimization process. In the real world ants communicate with each other indirectly, through a method known as stigmergy, to communicate whether their choices were beneficial or not. They lay down a chemical known as pheromone. The amount of pheromone laid down is dependent on how beneficial that path was for the ant that traveled down it. These

pheromones can then serve as markers for other ants and act as a map for where 
to find the best food and resources. Because pheromones eventually wear off when deposited on the path, the best tunnels will become repaved with pheromones while the less optimal paths will disappear over time. This leads to the eventual conclusion that all the ants will follow the same optimized path until a better one becomes available in the future.

In ACO algorithms, artificial ants are generated which follow artificial paths through discrete or NP-hard optimization problems. These artificial ants will then continually update the pheromone levels of their paths so that later ants will know which paths are optimal. Traditionally, ACO has been applied to round-trip problems, like the Traveling Salesman Problem. In these problems, ants leave from a random node, and travel to every available node in the problem, and then arrive back at the node from which they started. Based on the cost of their trip, they will change the amount of pheromone deposited at every node. This is in effect the same way that ants behave in real life. That is, they leave the nest, find food, and return to the nest later while other ants can access the route that they've taken. This methodology does not map directly over to other NP-hard problems, such as the orbit optimization problems tackled in this work. In this work the algorithm instead has to be modeled as a one way problem where the same basic idea is applied. Ants travel from the first planet to the last, and based on how much their trip costs, they will then alter the levels of pheromone deposited. The nodes here represent the planets on the interplanetary trajectory at different time steps. 
Chapter 5

\section{LAMBERT'S PROBLEM}

\subsection{The Two Body Lambert's Problem}

Lambert's problem was previously referenced in the Introduction to Orbital Mechanics section. The methodology behind this problem is key in building the desired interplanetary trajectory. This section gives a more in depth methodology on how to solve the problem. In order to determine the trajectory of an orbiting body in the two body problem, all that needs to be known are the initial and final position vectors as well as the time taken in order to complete the trajectory. The velocity corresponding to the initial position can be solved for and this initial state can therefor be propagated numerically to any point in time [3].

The two body Lambert's solution is a geometric solution that requires an iteration or expansion in order to build the trajectory. The solution is built from Kepler's equations of motion and an initial set of universal variables can be calculated. There are a few different orbital solutions depending on whether the trajectory is clockwise or counter-clockwise and whether or not multiple revolutions will occur until the spacecraft arrives at its desired trajectory. The angle between the position vectors must first be calculated and is given by equation 5.1 .

$$
\Delta \theta=\arccos \left(\frac{\overrightarrow{r_{1}} \cdot \overrightarrow{r_{2}}}{\left\|\overrightarrow{r_{1}}\right\|\left\|\overrightarrow{r_{2}}\right\|}\right)
$$

This equation gives the angle for the shortest direction trajectory. If it is desired to obtain a trajectory that takes the longer way around in the opposite direction then $\Delta \theta$ must be calculated differently specified by equation 5.2 . 


$$
\Delta \theta=2 \pi-\arccos \left(\frac{\overrightarrow{r_{1}} \cdot \overrightarrow{r_{2}}}{\left\|\overrightarrow{r_{1}}\right\|\left\|\overrightarrow{r_{2}}\right\|}\right)
$$

From the desired $\Delta \theta$, the value of $\mathrm{A}$ can then be determined with equation 5.3.

$$
A=\frac{\sqrt{\left\|\overrightarrow{r_{1}}\right\|\left\|\overrightarrow{r_{2}}\right\|} \sin (\Delta \theta)}{\sqrt{1-\cos (\Delta \theta)}}
$$

Through an initial guess of $\mathrm{z}=0$ the Stumpff functions can be defined which are given below for an elliptical orbit which are given by equations 5.4-5.5.

$$
\begin{gathered}
C(z)=\frac{1-\cos (\sqrt{z})}{z} \\
S(z)=\frac{\sqrt{z}-\sin (\sqrt{z})}{z^{\frac{3}{2}}}
\end{gathered}
$$

These Stumpff functions can then be used to determine y, the universal variable $\chi$, as well as an estimated trajectory time $\Delta \mathrm{T}$ given by equations $5.6-5.8$.

$$
\begin{gathered}
y=\left\|\overrightarrow{r_{1}}\right\|+\left\|\overrightarrow{r_{2}}\right\|+\frac{A(z S(z)-1)}{\sqrt{C(z)}} \\
\chi=\frac{\sqrt{y}}{C(z)} \\
\Delta T=\frac{\chi^{3} S(z)+A \sqrt{y}}{\sqrt{\mu}}
\end{gathered}
$$


These universal variables can then be iterated through a Newton's root finder method or bisection method. Each iteration, a new $\mathrm{z}$ value is found through the desired method and the new universal variables are calculated. Once $\Delta \mathrm{T}$ matches the desired flight time within the desired tolerance, then the final set of universal variables can be found. Using these universal variables the Lagrange variables can then be calculated. The Lagrange variables can be seen in equations 5.9-5.12.

$$
\begin{gathered}
f=1-\frac{y}{\left\|\vec{r}_{1}\right\|} \\
g=\frac{A \sqrt{y}}{\sqrt{\mu}} \\
\dot{g}=1-\frac{y}{\left\|\vec{r}_{2}\right\|} \\
\dot{f}=\frac{f \dot{g}-1}{g}
\end{gathered}
$$

From the set of Lagrange variables, the initial and final velocities of the trajectory can then be found from equations 5.13-5.14.

$$
\begin{gathered}
\vec{v}_{i}=\frac{\vec{r}_{2}-f \vec{r}_{1}}{g} \\
\vec{v}_{f}=\dot{f} \vec{r}_{1}+\dot{g} \vec{v}_{1}
\end{gathered}
$$

These are then the velocity vectors needed in order to link the interplanetary trajectory together. If this method is used for every planet to planet transfer, then the 
$\Delta \mathrm{V}$ necessary for the cost function can be determined. This method is straightforward and computationally inexpensive which makes it work very well for evolutionary optimization schemes. However this particular solution only works under the case of the two body dynamics problem. This foundation from the two body problem is still necessary however as it is needed for the more intensive method with orbital perturbations.

\subsection{The Perturbed Lambert's Problem}

In order to include perturbations into the dynamics, the method from the two body problem must be improved upon. A new iterative process must be compounded with the already existing iterative process contained within the two body Lambert's problem. A known initial and final position as well as a transfer time are known as is the case for the two body Lambert's problem. First, a two body Lambert's solution is run with the positions and transfer time that are desired. This will give an initial and final velocity which will serve as an initial guess for the iterative process. Both the initial position vector and the guess for the initial velocity vector found through the two body Lambert's problem can now be used to determine the state transition matrix through Shepperd's Method [14].

\subsubsection{Shepperd's Method}

This initial velocity as well as position, time of flight, and specific angular momentum of the main gravitational body are then needed in order to develop a state transition matrix for the system. This state transition matrix is calculated by the use of Shepperd's method which also gives the final velocity and position based on the two body problem. Note these final values are not the values that will be given when perturbations are inputted. The method implemented within STOpS, as well as described 
here is from the summary section of Shepperd's work, "Universal Keplerian State Transition Matrix" [14]. The reader is encouraged to reference Shepperd's method which is conducted to compute the state transition matrix as follows:

$$
u=0
$$

Here $\mathrm{u}$ is an initial guess that will be used as the first input in Kepler's iteration loop. $\beta$ can then be calculated which represents the energy of the orbit with equation 5.16 .

$$
\beta=\frac{2 \mu}{\left\|\vec{r}_{i}\right\|}-\left\|\vec{v}_{i}\right\|^{2}
$$

where $\mu$ is the standard gravitational parameter and the subscript i denotes the initial values of the trajectory. If $\beta$ is less than zero then $\Delta u=0$. If $\beta$ is greater than zero then $\Delta \mathrm{u}$ is defined by equations $5.17-5.19$

$$
\begin{gathered}
p=\frac{2 \pi \mu}{\beta^{\frac{3}{2}}} \\
n=\frac{T}{p}-\frac{2 \vec{r}_{i} \cdot \vec{v}_{i}}{p \beta}+\frac{1}{2} \\
\Delta u=\frac{2 n \pi}{\beta^{\frac{5}{2}}}
\end{gathered}
$$

where $\mu$ is the standard gravitational parameter and $\mathrm{T}$ is the time of flight of the trajectory. It is next necessary to enter Kepler's iteration loop. If convergence between true trajectory time $(T)$ and calculated trajectory time $(t)$ has not been met 
to within the specified tolerance, then the loop will repeat until tolerance is met. Kepler's iteration loop can be expressed by equations 5.20-5.30.

$$
\begin{aligned}
& q=\frac{\beta u^{2}}{1+\beta u^{2}} \\
& u_{0}=1-2 q \\
& u_{1}=2(1-q) u \\
& U=\frac{16}{15} u_{1}^{5} G\left(5,0, \frac{5}{2}, q\right)+\Delta U \\
& U_{0}=2 u_{0}^{2}-1 \\
& U_{1}=2 u_{0} u_{1} \\
& U_{2}=2 u_{1}^{2} \\
& U_{3}=\beta U+\frac{1}{3} U_{1} U_{2} \\
& r=\left\|\vec{r}_{i}\right\| U_{0}+\left\|\vec{v}_{i}\right\| U_{1}+\mu U_{2} \\
& t=\left\|\vec{r}_{i}\right\| U_{1}+\left\|\vec{v}_{i}\right\| U_{2}+\mu U_{3}
\end{aligned}
$$




$$
u_{n+1}=u_{n}-\frac{t-T}{4(1-q) r}
$$

where $\mathrm{G}$ is the continued fraction that must then be computed through the iterative process described by equations 5.31-5.44. The process must be continued until G converges.

$$
k=-9
$$

$$
l=3
$$

$$
d=15
$$

$$
n=0
$$

$$
A=1
$$

$$
B=1
$$

$$
G=1
$$

$$
k=-k
$$




$$
\begin{gathered}
l=l+2 \\
d=d+4 l \\
n=n+(1+k) l \\
A=\frac{d}{d-n A q} \\
B=(A-1) B \\
G_{n+1}=G_{n}+B
\end{gathered}
$$

The Lagrange variables can then be computed from the previously solved for values through the equations 5.45-5.48.

$$
\begin{gathered}
f=1-\left(\frac{\mu}{\left\|\vec{r}_{i}\right\|}\right) U_{2} \\
g=\left\|\vec{r}_{i}\right\| U_{1}+\left\|\vec{v}_{i}\right\| U_{2} \\
F=-\frac{\mu U_{1}}{r\left\|\vec{r}_{i}\right\|} \\
G=1-\left(\frac{\mu}{r}\right) U_{2}
\end{gathered}
$$


These Lagrange variables can then be used to calculate the desired positions and velocities through equations 5.49-5.50.

$$
\begin{gathered}
\vec{r}_{f}=f \vec{r}_{i}+g \vec{v}_{i} \\
\vec{v}_{f}=F \vec{r}_{i}+G \vec{v}_{i}
\end{gathered}
$$

Finally the state transition matrix can be found through the equations 5.51-5.57.

$$
\begin{aligned}
& W=g U_{2}+3 \mu U \\
& M=\left[\begin{array}{ccc}
\left(\frac{U_{0}}{r\left\|\vec{r}_{i}\right\|}+\frac{1}{\left\|\vec{r}_{i}\right\|^{2}}+\frac{1}{r^{2}}\right) F-\frac{\mu^{2} W}{r^{3}\left\|\vec{r}_{i}\right\|^{3}} & \frac{F U_{1}}{r}+\frac{G-1}{r^{2}} & \frac{(G-1) U_{1}}{r}-\frac{\mu}{r^{3}} W \\
-\frac{F U_{1}}{\left\|\vec{r}_{i}\right\|}-\frac{f-1}{\left\|\vec{r}_{i}\right\|^{2}} & -F U_{2} & -(\mathrm{G}-1) \mathrm{U}_{2} \\
\frac{(f-1) U_{1}}{\left\|\vec{r}_{i}\right\|}-\frac{\mu}{\left\|\vec{r}_{i}\right\|^{3}} W & (f-1) U_{2} & g U_{2}-W
\end{array}\right] \\
& \Theta_{11}=f I+\left[\begin{array}{lll}
\vec{r}_{f} & \vec{v}_{f}
\end{array}\right]\left[\begin{array}{ll}
M_{21} & M_{22} \\
M_{31} & M_{32}
\end{array}\right]\left[\begin{array}{ll}
\vec{r}_{i} & \vec{v}_{i}
\end{array}\right]^{T} \\
& \Theta_{12}=g I+\left[\begin{array}{cc}
\vec{r}_{f} & \vec{v}_{f}
\end{array}\right]\left[\begin{array}{cc}
M_{22} & M_{23} \\
M_{32} & M_{33}
\end{array}\right]\left[\begin{array}{lll}
\vec{r}_{i} & \vec{v}_{i}
\end{array}\right]^{T} \\
& \Theta_{21}=F I-\left[\begin{array}{ll}
\vec{r}_{f} & \vec{v}_{f}
\end{array}\right]\left[\begin{array}{ll}
M_{11} & M_{12} \\
M_{21} & M_{22}
\end{array}\right]\left[\begin{array}{ll}
\vec{r}_{i} & \vec{v}_{i}
\end{array}\right]^{T}
\end{aligned}
$$




$$
\begin{gathered}
\Theta_{22}=G I-\left[\begin{array}{cc}
\vec{r}_{f} & \vec{v}_{f}
\end{array}\right]\left[\begin{array}{cc}
M_{12} & M_{13} \\
M_{22} & M_{23}
\end{array}\right]\left[\begin{array}{ll}
\vec{r}_{i} & \vec{v}_{i}
\end{array}\right]^{T} \\
\Theta=\left[\begin{array}{cc}
\Theta_{11} & \Theta_{12} \\
\Theta_{21} & \Theta_{22}
\end{array}\right]
\end{gathered}
$$

where I is the 3X3 identity matrix. This state transition matrix is denoted by equation 5.57 and will be used after the propagation of our state matrix.

\subsubsection{Runge Kutta Fehlburg 78}

The initial state which is determined by the initial position vector and the guess for the initial velocity vector found through the two body Lambert's problem are then fed into a Runge Kutta 78 function. In this function, the number of equations being propagated, time of flight, step size, and truncation error tolerance must also be specified. The dynamics can then be incorporated from the perturbations and propagated by this Runge Kutta 78 function for the desired transfer time. An outline of the Runge Kutta Fehlberg 78 process is detailed in this section. This function is adapted from a Mathworks file exchange developed by David Eagle [5].This method is of the order of $O\left(h^{7}\right)$ with an embedded $8^{\text {th }}$-order method for step size control and a total of 13 stages. An initial guess for the step size is determined by input, $h$. This step size is continually refined with each iteration. The method also requires the user to input an acceptable truncation error tolerance. The matrices $\alpha, \beta$, and $\chi$ are constant integration coefficients specified by the Runge-Kutta 78 method. These integration coefficients are specified by equations 5.58-5.60. 


$\alpha=\left[\begin{array}{c}0 \\ 2 / 27 \\ 1 / 9 \\ 1 / 6 \\ 5 / 12 \\ 1 / 2 \\ 5 / 6 \\ 1 / 6 \\ 2 / 3 \\ 1 / 3 \\ 1 \\ 0 \\ 1\end{array}\right]$




$$
\beta=\left[\begin{array}{cccccccccccc}
0 & 0 & 0 & 0 & 0 & 0 & 0 & 0 & 0 & 0 & 0 & 0 \\
\frac{2}{27} & 0 & 0 & 0 & 0 & 0 & 0 & 0 & 0 & 0 & 0 & 0 \\
\frac{1}{36} & \frac{1}{12} & 0 & 0 & 0 & 0 & 0 & 0 & 0 & 0 & 0 & 0 \\
\frac{1}{24} & 0 & \frac{1}{8} & 0 & 0 & 0 & 0 & 0 & 0 & 0 & 0 & 0 \\
\frac{5}{12} & 0 & \frac{-25}{16} & \frac{25}{16} & 0 & 0 & 0 & 0 & 0 & 0 & 0 & 0 \\
\frac{1}{20} & 0 & 0 & \frac{1}{4} & \frac{1}{5} & 0 & 0 & 0 & 0 & 0 & 0 & 0 \\
\frac{-25}{108} & 0 & 0 & \frac{125}{108} & \frac{-65}{27} & \frac{125}{54} & 0 & 0 & 0 & 0 & 0 & 0 \\
\frac{31}{300} & 0 & 0 & 0 & \frac{61}{225} & \frac{-2}{9} & \frac{13}{900} & 0 & 0 & 0 & 0 & 0 \\
2 & 0 & 0 & \frac{-53}{6} & \frac{704}{45} & \frac{-107}{9} & \frac{67}{90} & 3 & 0 & 0 & 0 & 0 \\
\frac{-91}{108} & 0 & 0 & \frac{23}{108} & \frac{-976}{135} & \frac{311}{54} & \frac{-19}{60} & \frac{17}{6} & \frac{-1}{12} & 0 & 0 & 0 \\
\frac{2383}{4100} & 0 & 0 & \frac{-341}{164} & \frac{4496}{1025} & \frac{-301}{82} & \frac{2133}{4100} & \frac{45}{82} & \frac{45}{164} & \frac{18}{41} & 0 & 0 \\
\frac{3}{205} & 0 & 0 & 0 & 0 & \frac{-6}{41} & \frac{-3}{205} & \frac{-3}{41} & \frac{3}{41} & \frac{6}{41} & 0 & 0 \\
\frac{-1777}{4100} & 0 & 0 & \frac{-341}{164} & \frac{4496}{1025} & \frac{-289}{82} & \frac{2193}{4100} & \frac{51}{82} & \frac{33}{164} & \frac{12}{41} & 0 & 1
\end{array}\right]
$$




$$
\chi=\left[\begin{array}{c}
0 \\
0 \\
0 \\
0 \\
0 \\
34 / 105 \\
9 / 35 \\
9 / 35 \\
9 / 280 \\
9 / 280 \\
0 \\
41 / 840 \\
41 / 840
\end{array}\right]
$$

The derivatives of the states are calculated for the initial time from the equations of motion which include both the spacecraft's dynamics as well as the planet's equations of motion around the Sun. The first subsection of an $\mathrm{f}$ matrix can then be constructed from the derivatives of the states.

$$
f_{1: 6,1}=\dot{x}
$$

Starting with the first iteration, $d t_{n}$ is equal to h. Subsequent iterations will change this value. The subscript $\mathrm{k}$ denotes the dimensions of sub-matrices calculated from $\alpha$ and $\beta$. A new state is then iterated from the previous state. Starting with 
$\mathrm{k}=2$ the iterative process begins with equation 5.62 .

$$
x(i)_{f}=x(i)_{o}+d t_{n} \beta_{k, 1: k-1} f_{i, 1: k}
$$

This equation must be calculated for every variable in the state denoted by i to determine the entirety of the state. An updated time can then be determined from the $\alpha$ matrix and the relevant time step.

$$
t_{f}=t_{o}+\alpha_{k} d t_{n}
$$

The derivatives of the states are then calculated with the most recently calculated state and time with the specified equations of motion. The $\mathrm{f}$ matrix can then continue to be constructed with by equation 5.64 .

$$
f_{1: 6, k}=\dot{x}_{k}
$$

This process is repeated up to and including $\mathrm{k}=13$. Finally the whole $\mathrm{f}$ matrix has been constructed and the state can be propagated forward in time.

$$
x(i)_{n+1}=x(i)_{n}+d t_{n} \chi f_{i, 1: 13}
$$

This equation must be calculated for every variable in the state denoted by i. The truncation error can be calculated next by equation 5.66. 


$$
t_{e r}=\left|\left(f_{i, 1}+f_{i, 11}-f_{i, 12}-f_{i, 13}\right) \chi_{12} d t_{n}\right|
$$

This truncation error is then scaled by a tolerance where $t_{\text {etol }}$ is the truncation error tolerance inputted by the user.

$$
t_{\text {const }}=\frac{t_{\text {er }}}{|x(i)| t_{\text {etol }}+t_{\text {etol }}}
$$

If the scaled truncation error is greater than the truncation error tolerance then the state error is set to the scaled truncation error. If the truncation error tolerance is larger than the scaled truncation error then the state error is set equal to the truncation error tolerance. Finally an updated step size can be calculated from the state error.

$$
d t_{n+1}=0.8 d t_{n}\left(\frac{1}{x_{e r r}}\right)^{\frac{1}{8}}
$$

This iterative process is continued until the final time and position is then reached. This will give a final position vector at the end of the trajectory.

\subsubsection{Determination of the True Trajectory}

This final position vector found by propagating using Runge Kutta 78 will not match the position of the desired planet arrival position yet. Instead the norm of the difference of these positions must be calculated which is expressed by equation 5.69.

$$
\|\Delta \vec{r}\|=\left\|\vec{r}_{f}-\vec{r}_{r k}\right\|
$$


Where the subscript $\mathrm{f}$ denotes the true final position vector and rk denotes the final position vector propagated by Runge Kutta 78. This difference in final position will be used to determine when the iterative process will end. Initially $\|\Delta \vec{r}\|$ will be large but through the iterative process it will shrink to be within the allowable tolerance set by the user and the iterative process will then end. From this $\Delta \vec{r}_{f}$, a $\Delta \mathrm{V}$ correction vector must then be calculated through equation 5.70.

$$
\Delta \vec{v}_{c}=\Theta^{-1}\left(\Delta \vec{r}_{f}\right)^{T}
$$

This $\Delta V$ correction vector is then added to the initial velocity vector found from the two body Lambert problem to give an updated initial velocity vector given by equation 5.71.

$$
\vec{v}_{i(n+1)}=\vec{v}_{i(n)}+\Delta \vec{v}_{c}
$$

This process is repeated to find a more accurate state transition matrix until the $\left\|\Delta \vec{r}_{f}\right\|$ value is within its required tolerance. Once the iterative process is finished then the correct initial velocity for the Lambert's trajectory with perturbations has been obtained. This initial position and velocity can then be propagated one more time with Runge Kutta Fehlberg 78 with the desired equations of motion to find the true final velocity of the Lambert's problem.

\subsubsection{Consideration for Lambert's Perturbations}

If it is desired to include n-body perturbations into the analysis, that is the gravitational effects from other planets, then the positions and velocities of these planets must be included into the states. Because it is desired that multiple planets be added, 
and because the gravitational force from these planets create the dominant magnitude of acceleration on the spacecraft, it is important that they be included. It is assumed for the sake of this analysis that the planets themselves are not under the influence of any perturbations but instead only have their accelerations derived from the Sun's gravity. Although the exact positions of the planets will vary slightly from the propagated positions, the change in acceleration that such a difference would make is considered negligible. For example the largest change in the semi-major axis which will occur due to perturbations for any of the planets is Saturn. The change in distance accumulated over one century is just $0.003 \mathrm{AU}$ while the changes for the other planets are even smaller [16].

It also needs to be noted that even without the addition of the states from the planets that this iterative process is far more computationally expensive than the two body Lambert's problem because there must be multiple iterations before the system converges to the correct values and because each state has to be propagated using the Runge Kutta 78 function. With the addition of these planet states, STOpS will take a significantly longer amount of time to find a solution. This is due to the nature of evolutionary optimization algorithms that because they have a population of trajectory and many generations of those populations, the number of times that the perturbed Lambert's problem is called can quickly add up to the order of thousands of simulations. 
Chapter 6

\section{GRAVITATIONAL ASSISTS}

\subsection{The Two Body Gravity Assist}

The flyby or gravity assist section of the code is in STOpS to ensure that a spacecraft can obtain a large change in its velocity vector without expending any of its own fuel. These $\Delta V$ maneuvers help the spacecraft reach destinations it otherwise wouldn't be able to. As discussed earlier these gravity assist maneuvers are modeled as hyperbolic trajectories in the flyby body's reference frame. A diagram of such a trajectory can be seen in figure 6.1 and the values listed in the figure will later be calculated in order to determine the correct trajectory.

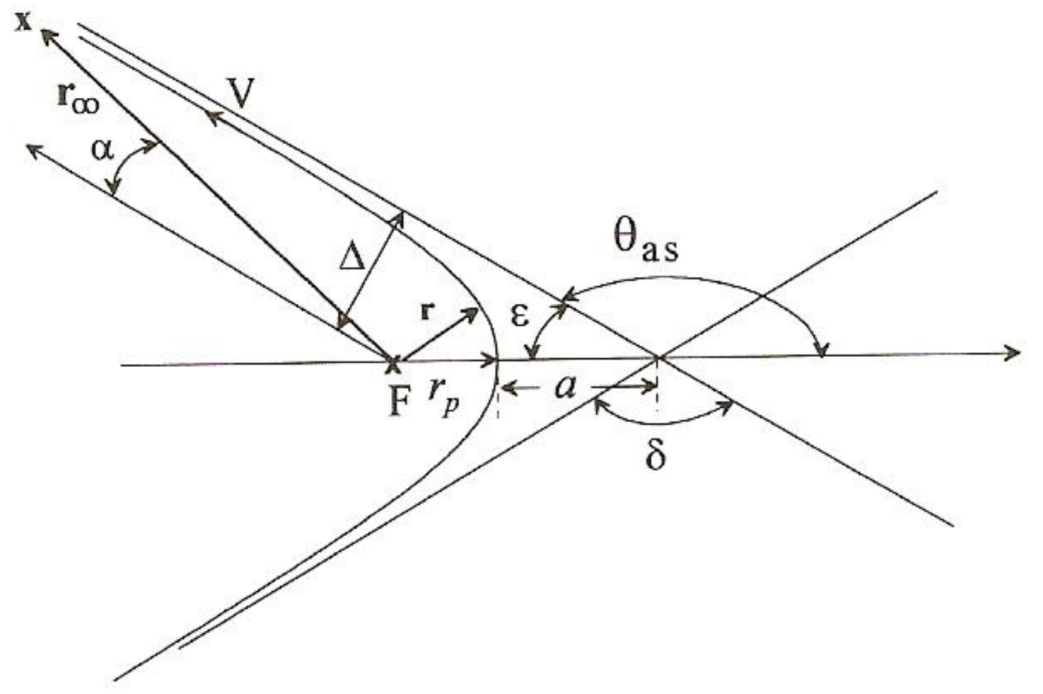

\section{Figure 6.1: Hyperbolic Gravity Assist Maneuver}

Because the Lambert's solver links together a collection of trajectories from planet to planet, the magnitude of the initial and final velocity for the flyby will not necessarily be equal. It is the case that under only the influence of the gravitational assist 
body, the magnitudes of the initial and final velocity vectors will remain constant. Because of this the extra $\Delta \mathrm{V}$ needed must be supplied through the spacecraft's thruster. Because it is not beneficial for the spacecraft to use fuel during these gravity assists, the optimization process should be expected to drive the difference in the magnitudes of the initial and final velocities to a smaller value over successive generations. The velocity expended by the spacecraft during this flyby is expressed by the equation 6.1 .

$$
\Delta V=\left|\left\|\vec{v}_{\infty 2}\right\|-\left\|\vec{v}_{\infty 1}\right\|\right|
$$

where $\vec{v}_{\infty 2}$ is the velocity needed at the end of the hyperbolic trajectory and $\vec{v}_{\infty 1}$ is the initial velocity at the start of the hyperbolic trajectory. In the two body gravity assist problem there were then some assumptions that had to be made in order to make it compatible with STOpS. For example, the radius of perigee of the hyperbolic orbit was allowed to fall within the radius of the flyby planet. This is not realistic to a true trajectory as this would cause the spacecraft to crash on the planets surface but it was desired to preserve these trajectories based on this problem. Instead if the flyby were to fall inside the radius of the planet, then a flat $\Delta \mathrm{V}$ penalty would be added to these trajectories. Another flat $\Delta \mathrm{V}$ penalty was added to any trajectory that would pass within the planets atmosphere. These flat $\Delta \mathrm{V}$ penalties are given below by equations $6.2-6.3$.

$$
\begin{gathered}
\Delta V_{1}=\frac{r_{\min }}{r_{p}} \\
\Delta V_{2}=3\left(\frac{R}{r_{p}}\right)
\end{gathered}
$$


where $r_{\min }$ is the radius extending to the end of the planet's atmosphere, $\mathrm{R}$ is the radius of the planet, and $r_{p}$ is the radius of perigee of the hyperbolic trajectory. Both of these penalties will be replaced with a more realistic model when perturbations are later added into the system. Because this code utilizes patch conics then there needs to be a cut off position to where the dominant acceleration on the spacecraft is coming from the planet rather than the Sun.

The sphere of influence was chosen for this initial and final position for the start of propagation of the flyby trajectory. The sphere of influence is defined as the point where the magnitude of the acceleration from one body becomes larger than another body. In this case where the acceleration from the planet becomes larger than the acceleration from the Sun. The sphere of influence can be expressed by the equation 6.4 .

$$
R_{S O I}=a\left(\frac{m}{M}\right)^{\frac{2}{5}}
$$

where $\mathrm{m}$ is the mass of the smaller gravitational body which in this case are the planets, $\mathrm{M}$ is the mass of the larger gravitational body which in this case is the Sun, and $\mathrm{a}$ is the semi major axis of the smaller gravitational body's orbit around the larger gravitational body.

\subsection{The Perturbed Gravity Assist}

In the updated version of STOpS these trajectories will be ruled out as they are fundamentally unrealistic to the spacecraft environment. However perturbations will also produce a force on the spacecraft which will in fact change the initial velocity of the flyby so that its magnitude will not match the final velocity. Because of this it is 
necessary to propagate the initial position and velocity at the start of the flyby with perturbation dynamics to see just how much this final velocity vector will change by the time it reaches its final position.

As seen from equation 6.4, it is simple to find the magnitude of the sphere of influence. Because of the desire to propagate the spacecraft's trajectory from the sphere of influence, there needs to be a specific position vector whose magnitude matches that of the sphere of influence. Because the only input into the system that is the initial velocity derived from the Lambert trajectory from one planet to another, it is difficult then to derive the initial position that is needed. Because of the nature of a hyperbolic orbit, the position vector is defined from the center of the planet to the spacecraft. The velocity vector of the spacecraft will be tangent to the trajectory of the hyperbola. At $v_{\infty}$ the velocity vector will point directly at the origin. Because the sphere of influence is at a large distance from the planet then the assumption will be made that the spacecraft has an initial velocity vector which matches $\vec{v}_{\infty}$. At the sphere of influence then the direction of the velocity vector should be close to exactly opposite the position vector although depending on the distance to the sphere of influence these directions will vary somewhat. In order to calculate the exact initial position vector some necessary values must be known. The first values that must be found are $\delta$ known as the turn angle, the eccentricity of the hyperbolic orbit, the radius of perigee of the orbit, and the distance to the focus point which is also the location of the planet. The method that follows is used to determine this needed position vector. Refer to the hyperbolic diagram in figure 6.1 for angles and values needed. These parameters are given by equations 6.5-6.8.

$$
\delta=\arccos \left(\frac{\vec{v}_{1} \cdot \vec{v}_{2}}{\left\|\vec{v}_{1}\right\|\left\|\vec{v}_{2}\right\|}\right)
$$




$$
\begin{gathered}
e=\frac{1}{\sin (\delta / 2)} \\
r_{p}=\frac{\mu(e-1)}{\left\|\vec{v}_{1}\right\|^{2}} \\
a=\frac{r_{p}}{1-\frac{1}{e}}
\end{gathered}
$$

The next values that need to be calculated are $\beta, \gamma, \theta, \alpha_{1}$, and $\alpha_{2}$. These angles can be seen in the hyperbolic diagram. They can be all be calculated from the first set of calculated values. These values are given by equations 6.9-6.13 where all values are measured in radians.

$$
\begin{gathered}
\beta=\arcsin \left(\frac{a * \sin \left(\frac{\pi-\delta}{2}\right)}{R_{S O I}}\right) \\
\gamma=\frac{\pi}{2}-\beta+\frac{\delta}{2} \\
\theta=2 \pi-2 \gamma \\
\alpha_{1}=\theta-\frac{\pi}{2}+\frac{\delta}{2} \\
\alpha_{2}=-\alpha_{1}
\end{gathered}
$$

The unit vectors associated with the $v_{\infty}$ vectors are also needed. These can simply be found by taking the vectors and dividing them by their associated norms. 


$$
\begin{gathered}
\hat{v}_{\infty 1}=\frac{\vec{v}_{\infty 1}}{\left\|\vec{v}_{\infty 1}\right\|} \\
\hat{v}_{\infty 2}=\frac{\vec{v}_{\infty 2}}{\left\|\vec{v}_{\infty 2}\right\|}
\end{gathered}
$$

A unit vector that is perpendicular to both unit $v_{\infty}$ vectors must be calculated next. This can simply be calculated by finding the cross product between the two unit $v_{\infty}$ vectors expressed by equation 6.16 .

$$
\hat{w}=\frac{\hat{v}_{\infty 2} \times-\hat{v}_{\infty 1}}{\left\|\hat{v}_{\infty 2} \times-\hat{v}_{\infty 1}\right\|}
$$

It is known that both the velocity vectors and position vectors lie in the same plane. Because of this it is then possible to find some rotation of the $v_{\infty}$ vectors about $\hat{v}_{3}$ to calculate both unit position vectors represented by the direction of the initial and final positions. These unit position vectors can therefor be calculated by equations 6.17-6.18 which are produced from a rotation matrix [1]. All vectors and vector components in this equation are from the unit vectors $\hat{v}_{\infty 1}, \hat{v}_{\infty 2}$, and $\hat{w}$.

$$
\begin{gathered}
\hat{r}_{i}=\left[\begin{array}{l}
-w_{x}\left(\left(\hat{v}_{\infty 1} \cdot \hat{w}\right)\left(1-\cos \left(\alpha_{1}\right)\right)\right)+\left(w_{z} v_{\infty 1 y}-w_{y} v_{\infty 1 z}\right) \sin \left(\alpha_{1}\right) \\
-w_{y}\left(\left(\hat{v}_{\infty 1} \cdot \hat{w}\right)\left(1-\cos \left(\alpha_{1}\right)\right)\right)+\left(w_{x} v_{\infty 1 z}-w_{z} v_{\infty 1 x}\right) \sin \left(\alpha_{1}\right) \\
-w_{z}\left(\left(\hat{v}_{\infty 1} \cdot \hat{w}\right)\left(1-\cos \left(\alpha_{1}\right)\right)\right)+\left(w_{y} v_{\infty 1 x}-w_{x} v_{\infty 1 y}\right) \sin \left(\alpha_{1}\right)
\end{array}\right] \\
\hat{r}_{f}=\left[\begin{array}{l}
-w_{x}\left(\left(-\hat{v}_{\infty 2} \cdot \hat{w}\right)\left(1-\cos \left(\alpha_{2}\right)\right)\right)+\left(w_{y} v_{\infty 2 z}-w_{z} v_{\infty 2 y}\right) \sin \left(\alpha_{2}\right) \\
-w_{y}\left(\left(-\hat{v}_{\infty 2} \cdot \hat{w}\right)\left(1-\cos \left(\alpha_{2}\right)\right)\right)+\left(w_{z} v_{\infty 22}-w_{x} v_{\infty 2 z}\right) \sin \left(\alpha_{2}\right) \\
-w_{z}\left(\left(-\hat{v}_{\infty 2} \cdot \hat{w}\right)\left(1-\cos \left(\alpha_{2}\right)\right)\right)+\left(w_{x} v_{\infty 2 y}-w_{y} v_{\infty 2 x}\right) \sin \left(\alpha_{2}\right)
\end{array}\right]
\end{gathered}
$$


These unit vectors are then multiplied by the sphere of influence in order to obtain the initial and final position vectors for the flyby which are given by equations 6.196.20 .

$$
\begin{gathered}
\vec{r}_{i}=R_{S O I} \hat{r}_{i} \\
\vec{r}_{f}=R_{S O I} \hat{r}_{f}
\end{gathered}
$$

Once the position vectors are known, the initial position can then be propagated to the final position to determine how the magnitude of the velocity vector has changed. If it is desired to propagate with the n-body perturbations included from the planets moons then it is also necessary to determine the orbital elements of the moons with respect to the planet as well as the correct location that the moons will be in given the desired flyby time. With all this information an accurate $\Delta \mathrm{V}$ can then be determined which is needed by the spacecraft in order to continue on the desired optimized trajectory. This $\Delta \mathrm{V}$ expended by the spacecraft can be expressed by equation 6.21 .

$$
\Delta V=\left|\left\|\vec{v}_{f}\right\|-\left\|\vec{v}_{\infty 2}\right\|\right|
$$

where $\left\|\vec{v}_{f}\right\|$ is the velocity at the final propagated position with perturbations in the dynamics and $\left\|\vec{v}_{\infty 2}\right\|$ is the exit velocity needed in order to maintain the trajectory towards the next target planet. From this the cost function has all of the information needed for this section of the trajectory. 
Chapter 7

RESULTS AND ANALYSIS

\subsection{Perturbation Magnitude Comparison}

In order to gain a deeper understanding on which factors are going to affect a spacecraft's trajectory the most, it is necessary to look at which perturbations carry the most influence over the spacecraft's motion and when these accelerations are most prominent. For example, the acceleration due to the gravitational force of Jupiter will dominate over the other environmental perturbations for many interplanetary trajectories. This analysis is also useful in determining which perturbations should be considered based on a particular mission. This includes considering which planetary accelerations should be included and which should be assumed negligible based on the magnitude of the acceleration. Because a Lambert's solution becomes computationally expensive with the addition of n-body accelerations, it may be deemed useful to omit certain planetary accelerations for the sake of time in order to evaluate the optimization process effectively. An evaluation of the strength of these perturbations at a distance of one astronomical unit is given by Table 7.1 in order to determine the influence that these perturbations have over the spacecraft. The accelerations from $J_{2}$, solar radiation pressure, and general relativity are measured at one astronomical distance from the Sun while the accelerations due to the planets are measured at one astronomical unit from the center of the corresponding planet. 


\section{Table 7.1: Perturbation Magnitude Comparison at One Astronomical Unit}

\begin{tabular}{|c|c|}
\hline & acceleration $\left(\mathrm{km} / \mathrm{s}^{2}\right)$ \\
\hline$J_{2}$ & $2.005 \mathrm{e}-13$ \\
\hline SRP & $2.302 \mathrm{e}-14$ \\
\hline General Relativity & $1.387 \mathrm{e}-13$ \\
\hline Mercury & $9.844 \mathrm{e}-13$ \\
\hline Venus & $1.452 \mathrm{e}-11$ \\
\hline Earth & $1.781 \mathrm{e}-11$ \\
\hline Mars & $1.914 \mathrm{e}-12$ \\
\hline Jupiter & $5.661 \mathrm{e}-9$ \\
\hline Saturn & $1.695 \mathrm{e}-9$ \\
\hline Uranus & $2.589 \mathrm{e}-10$ \\
\hline Neptune & $3.055 \mathrm{e}-10$ \\
\hline
\end{tabular}

\subsection{Analysis of Test Case Trajectory}

In order to obtain an understanding of the degree to which perturbations may affect a spacecraft on orbit it is desired to run a test case. This test case is focused on the trajectory from Earth with a flyby around Jupiter and an arrival at Saturn. An optimized trajectory was found for this mission without the presence of perturbations. STOpS was set to look for departure dates within a five year window between January 11995 and January 1 2000. The optimization process utilized the genetic algorithm, differential evolution algorithm, and the particle swarm algorithm. STOpS was able to find an optimized trajectory whose parameters can be viewed by Table 7.2. This trajectory was successful in arriving at the desired locations of Jupiter and Saturn 
within a respectable time frame. The spacecraft's trajectory, which includes the orbits of the planets as well as each leg of the trajectory can be seen in figure 7.1.
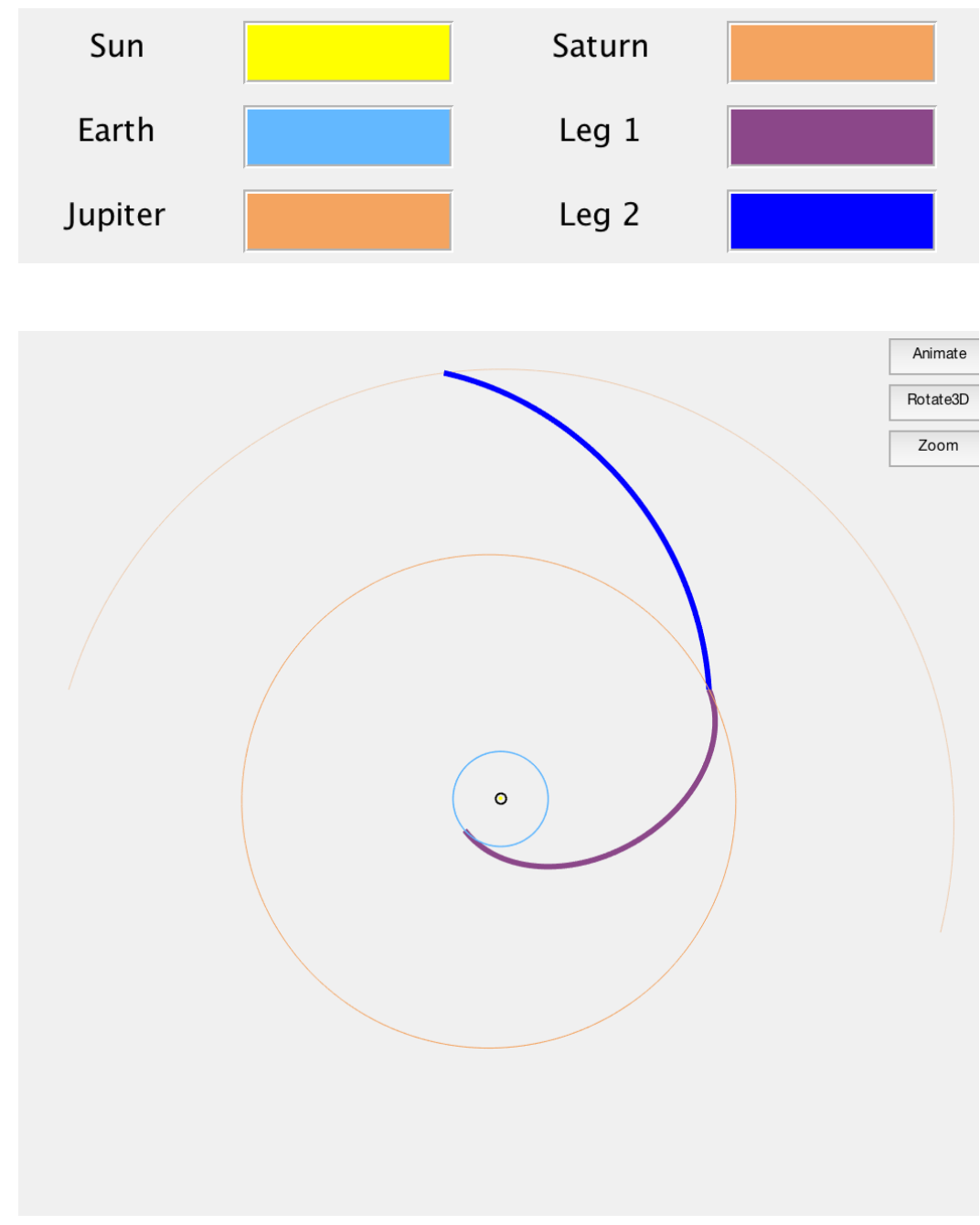

Figure 7.1: Trajectory From Earth to Saturn

Table 7.2: Flight Parameters of Trajectory From Earth to Saturn

\begin{tabular}{|c|c|c|}
\hline & Flight Times & $\Delta \mathrm{V}(\mathrm{km} / \mathrm{s})$ \\
\hline Earth & May 2 1997 & 9.4642 \\
\hline Jupiter & September 27 1999 & 0.0035 \\
\hline Saturn & November 5 2003 & 3.7811 \\
\hline
\end{tabular}


The initial values for this optimized trajectory were then taken and propagated with perturbations for the same time period. All other conditions of the orbit remained the same. The goal of this analysis was to determine how far off the final position vector would be from the perturbations when compared to the two body problem. When propagated for the time period needed to arrive at Jupiter of 877 days, it was determined that the magnitude of the difference in the position vectors was $2668000 \mathrm{~km}$. Although this would still leave us within the sphere of influence of Jupiter's orbit by the end of this trajectory, this is still a very large error distance if it is necessary to perform a flyby at a specific radius of perigee.

This analysis shows that because of perturbations the spacecraft can end up in a very different arrival destination if it is assumed that the only acceleration affecting the spacecraft is the gravity from the Sun. This has the potential to make the optimized trajectory useless because if the spacecraft misses its first flyby by a large distance then it will continue to drift off course and eventually these errors will compound to send it in a largely different direction than the trajectory that is desired. With the introduction of perturbations into the analysis there can be more certainty that the calculated orbit will send the spacecraft on the correct trajectory to meet all of the flybys at the correct radius of perigee.

\subsection{Cassini Trajectory}

\subsubsection{Cassini Background}

Cassini was a space probe launched in October 151997 whose destination was Saturn. The trajectory departed from Earth and then utilized two flybys around Venus before making another flyby around Earth. The trajectory continued on to Jupiter where it did its final flyby before arriving at Saturn on July 1 2004. The spacecraft burned one more time at the end of its trajectory in order to insert itself around Saturn in 
a parking orbit. Over the course of its lifetime Cassini collected valuable data on Saturn's rings, its atmosphere, and multiple different moons. This included sending a separate Huygens probe down to the surface of Titan to collect data on its surface and atmosphere. Cassini completed its mission by colliding with Saturn on September 15 $2017[15]$.

\subsubsection{Cassini Optimization Setup}

The trajectory panel was setup so that all of the flyby planets and the arrival planet of Saturn were specified. STOpS was given a two month window in order to look for launch dates between October 11997 and November 30 1997. This initial constraint had to be specified in order for the optimized trajectory to converge on a desirable solution. The trajectory panel can be viewed in figure 7.2. 


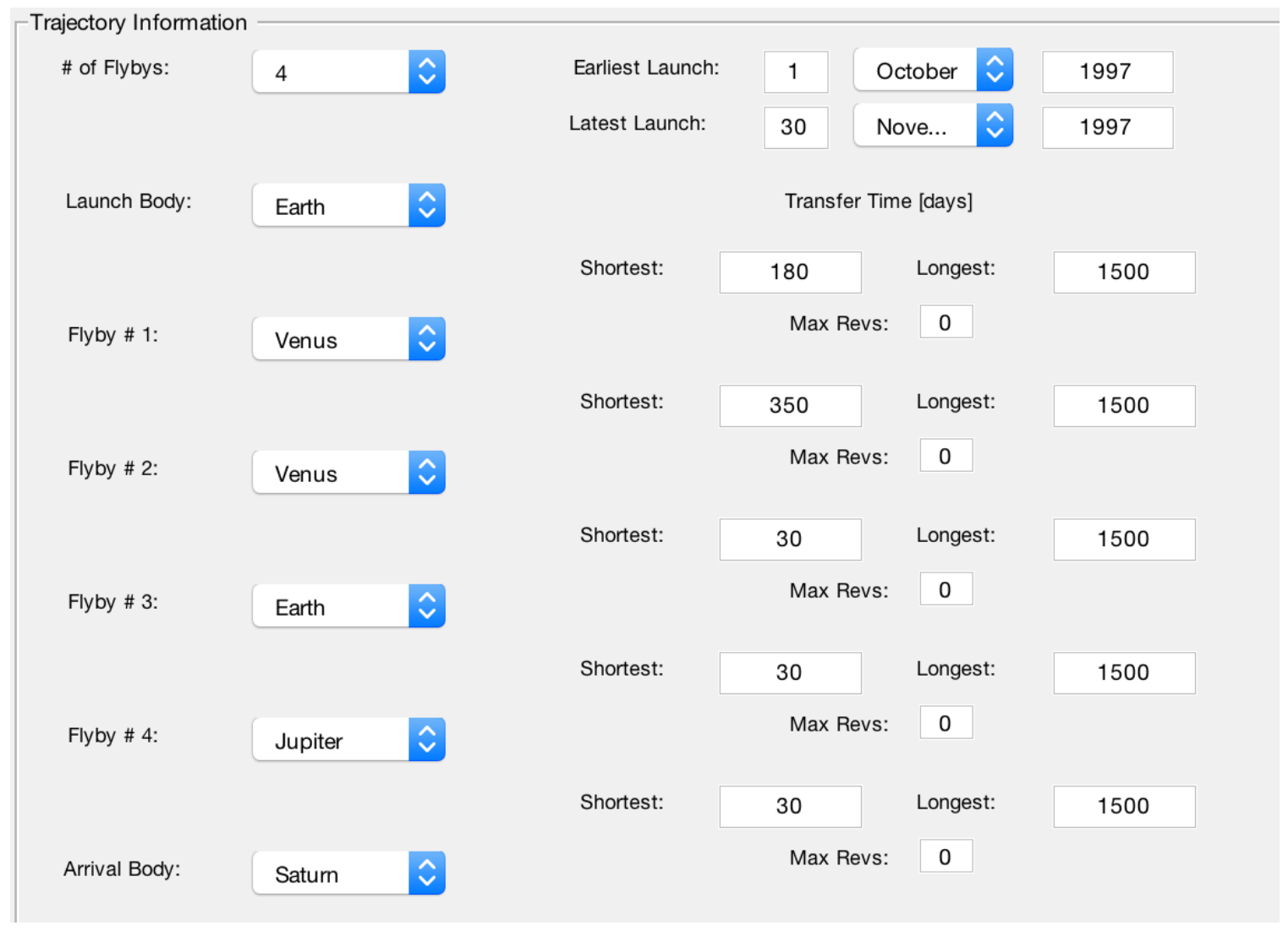

Figure 7.2: Cassini's Trajectory Panel

The cost function which was used for this trajectory, including the weights and parameters chosen can be viewed in figure 7.3. The weights were normalized so that the costs corresponding to each parameter were the same order of magnitude. This normalization is important to ensure that one parameter of the cost function will not dominate in importance over all of the other parameters. The arrival $\Delta \mathrm{V}$ at Saturn was included into the cost function because Cassini has to insert itself into the orbit of Saturn upon arrival and the difference in velocity should be low in order to obtain the optimal trajectory. Slightly more of an influence was put on the $\Delta \mathrm{V}$ weight in order to ensure that the trajectory was feasible based on fuel limitations for the Cassini mission. 


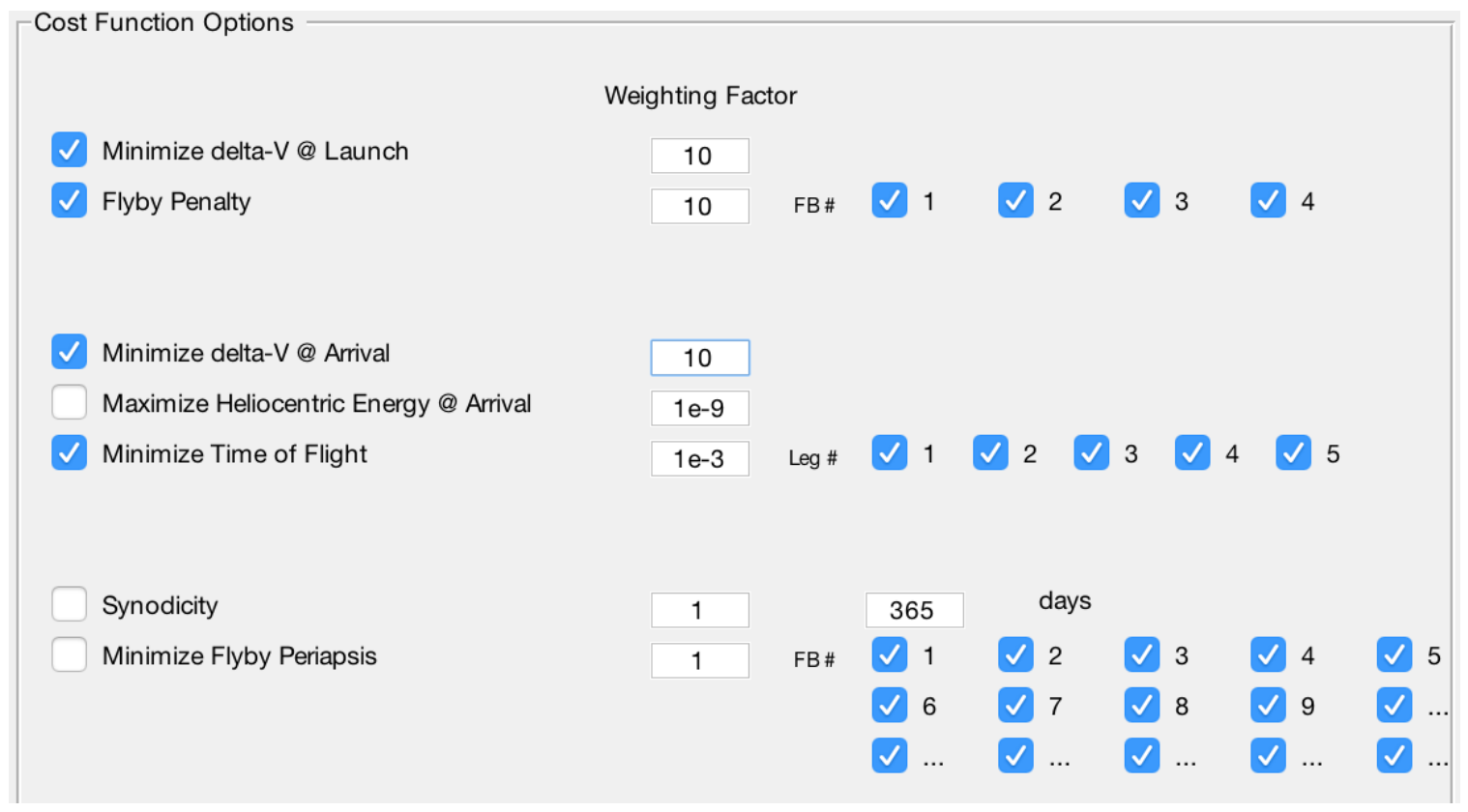

\section{Figure 7.3: Cassini's Cost Function Panel}

The algorithms included into this optimization were the genetic algorithm and the differential evolution algorithm. These two algorithms were used because the addition of the other algorithms produced no improvements while slowing the optimization process down. One migration was used and 30 generations were allocated to each algorithm. For the purpose of this analysis the population was set to 200 members. This proved to be large enough so that the same optimized solution could be found reliably and small enough so that the computational time remained at a feasible value. Also, the number of generations was base lined at 30 as by this point the cost becomes stagnant for most trajectories and no further improvements can be found. In order for these results to be repeatable, the population members and number of migrations should equal or exceed the values used here in order to ensure that the optimization process is given enough opportunity to converge on an optimal solution. It is recommended that the other parameters such as crossover probability, mutation probability, scaling factor, members to keep, and cross over points remain constant in order for STOpS to converge on an optimal trajectory. As discussed in the 
optimization chapter, if the crossover probability, mutation probability, and crossover point values are graded too highly then the optimization search will become far too random whereas if these values are too low, then the search will become stagnant and arrive at a non-optimal solution. This optimization panel can be seen in figure 7.4.

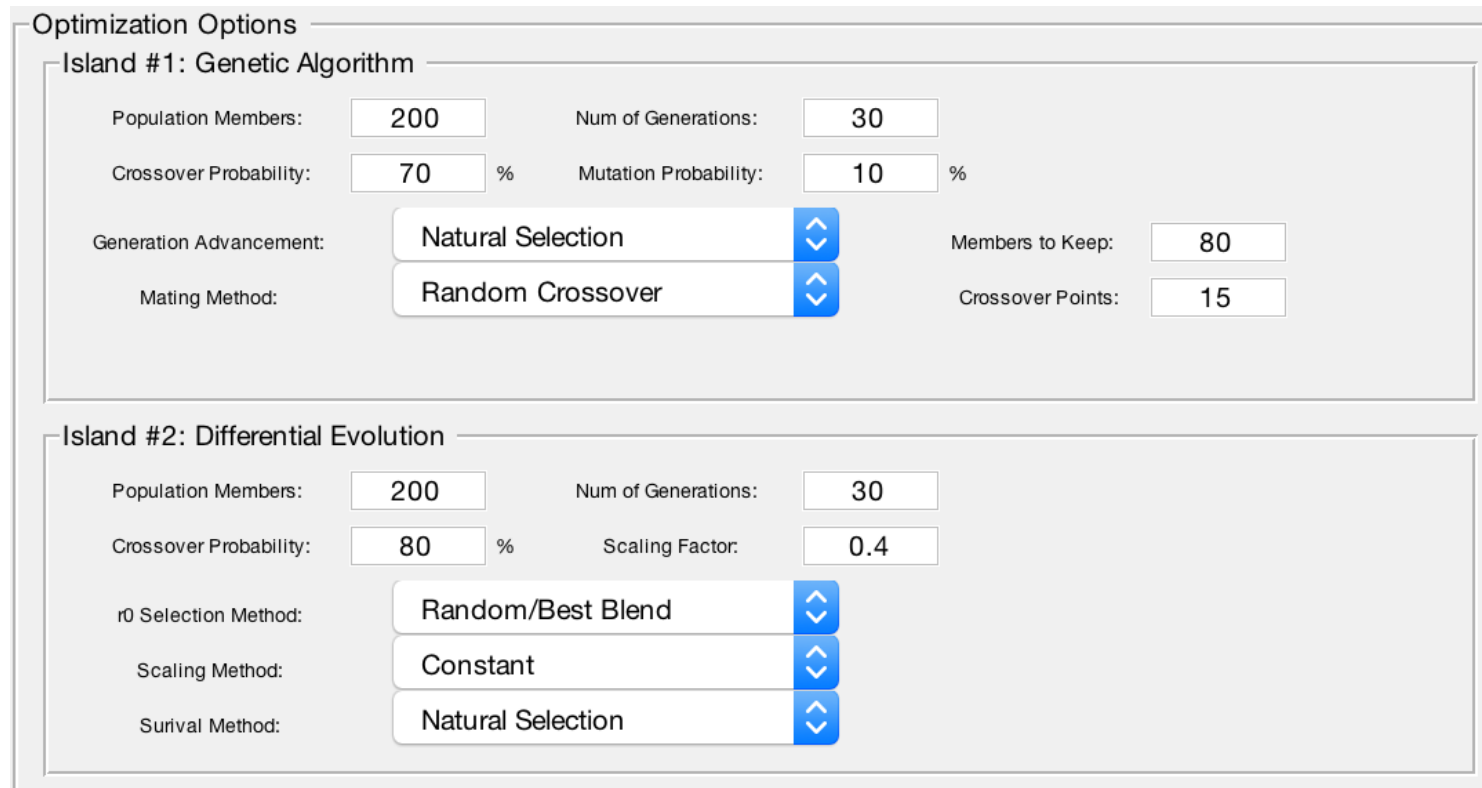

Figure 7.4: Cassini's Optimization Panel

\subsubsection{Cassini Analysis Without Perturbations}

When run without any perturbations, STOpS was able to calculate a trajectory which maintained similar times of flight for each leg of the trajectory when compared to the actual trajectory utilized by Cassini on its mission. For this analysis, Lambert's method was constrained so that all orbital transfers were in the prograde direction of the planets. This is due to the fact that many of the planetary transfers must take the long way option to be truly optimal for the Cassini mission. Also important is that any trajectories which fly retrograde to the motion of the planets require a very large amount of $\Delta \mathrm{V}$ in order to be completed. Therefor these retrograde trajectories 
are highly unlikely to be the optimal trajectory and can safely be ruled out of the optimization process. It should also be noted that the $\Delta \mathrm{V}$ at arrival is the difference in velocity between the spacecraft and Saturn at the end of the trajectory. While it is still important to minimize this velocity, only a fraction of this $\Delta \mathrm{V}$ needs to be utilized in order to insert itself into Saturn's orbit. For example in Cassini's actual trajectory although the difference in velocity between Saturn and the spacecraft was $5.3616 \mathrm{~km} / \mathrm{s}$, the $\Delta \mathrm{V}$ needed for orbital insertion was only $0.622 \mathrm{~km} / \mathrm{s} \mathrm{[15].} \mathrm{An}$ average of five optimized trajectories as well as the trajectory with the best cost out of the five optimized, is compared to the Cassini mission. The optimized trajectory with the lowest cost can be viewed in figure 7.5 in comparison to the actual Cassini trajectory in figure 7.6. The legend provides the orbits of the planets as well as each leg of the trajectory.

\begin{tabular}{|c|c|}
\hline Sun & Leg 1 \\
\hline Venus & Leg 2 \\
\hline Earth & Leg 3 \\
\hline Jupiter & Leg 4 \\
\hline Saturn & Leg 5 \\
\hline
\end{tabular}




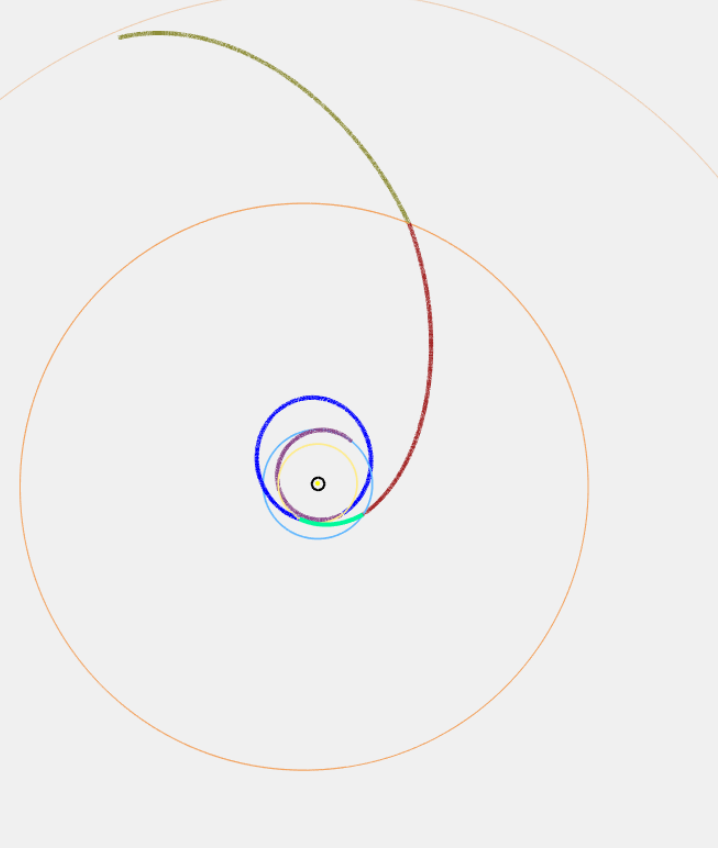

Figure 7.5: Cassini's Optimized Trajectory

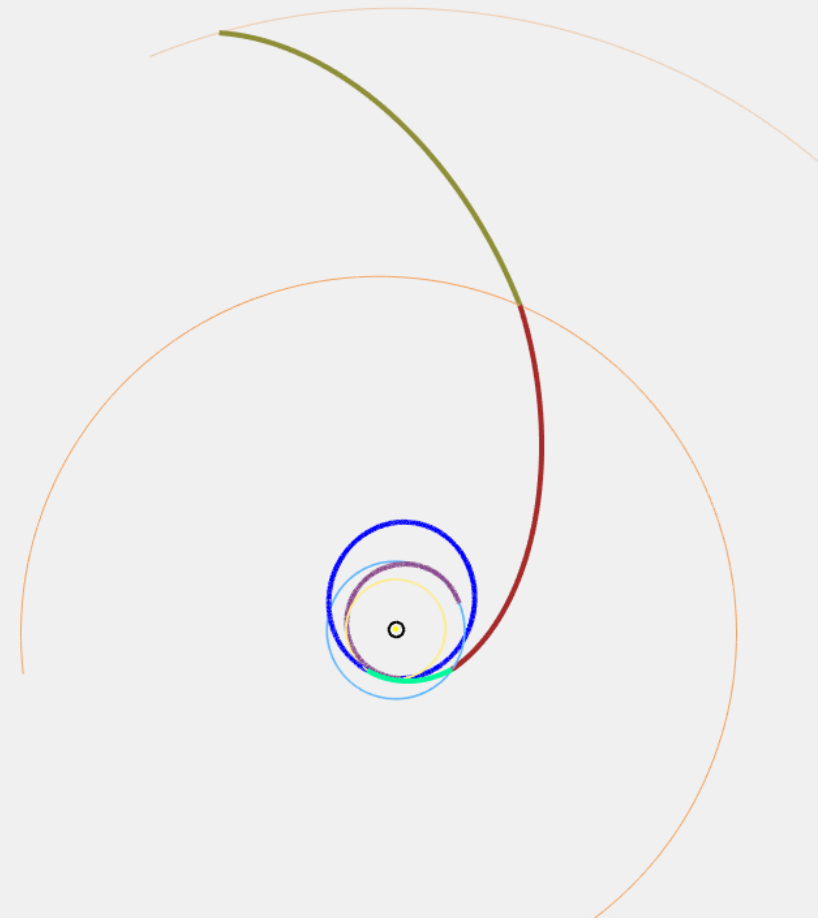

Figure 7.6: Actual Cassini Trajectory 
The analysis of the cost parameters for the trajectory can be viewed in Table 7.3. A list of flight dates and $\Delta \mathrm{V}$ expenditures are shown including all $\Delta \mathrm{V}$ values for flybys around each planet. The $\Delta \mathrm{V}$ values corresponding to the flyby portion of the trajectory are only from the change in velocity due to the spacecraft's thruster. The final cost of the trajectories are compared against each other here as well. The flight times for the best optimized trajectory as well as the actual trajectory are shown by Table 7.4. These flight times are within reason in comparison to the true trajectory and are able to create a successful trajectory.

Table 7.3: Cost Parameter Comparison for Cassini's Unperturbed Trajectory

\begin{tabular}{|c|c|c|c|}
\hline & Best Unperturbed & Average Unperturbed & Actual Trajectory \\
\hline$\Delta$ V Departure $(\mathrm{km} / \mathrm{s})$ & 3.9832 & 3.9947 & 3.8709 \\
\hline$\Delta \mathrm{V}$ Flyby $(\mathrm{km} / \mathrm{s})$ & 2.3825 & 3.1116 & 0.5489 \\
\hline$\Delta \mathrm{V}$ Arrival $(\mathrm{km} / \mathrm{s})$ & 5.1718 & 4.4684 & 5.3616 \\
\hline Total Cost & 117.8762 & 118.3492 & 100.265 \\
\hline
\end{tabular}




\section{Table 7.4: Flight Time Comparison for Cassini's Unperturbed Trajectory}

\begin{tabular}{|c|c|c|}
\hline & Best Unperturbed & Actual Trajectory \\
\hline Earth & November 191997 & October 151997 \\
\hline Venus & May 191998 & April 26 1998 \\
\hline Venus & June 261999 & June 24 1999 \\
\hline Earth & August 18 1999 & August 18 1999 \\
\hline Jupiter & January 11 2001 & December 302000 \\
\hline Saturn & September 24 2004 & July 1 2004 \\
\hline
\end{tabular}

When these optimized trajectories are compared with the actual Cassini trajectory, the flight times are very similar. For comparison the total flight time for the best optimized trajectory was 2501 days while the total flight time for the actual trajectory was 2451 days. This amounts to a 2 percent difference. However while the flight times for this trajectory are similar, the actual trajectory performed by Cassini achieves a much lower cost. This is a result of the actual Cassini mission performing a $\Delta \mathrm{V}$ maneuver in between the two Venus flybys rather than during them. As a result the real mission was able to use significantly less $\Delta \mathrm{V}$ during its time between departure from Earth and arrival at Saturn. Because STOpS is not currently capable of expending $\Delta \mathrm{V}$ in between flybys this led to the larger difference for this parameter of the trajectory. For comparison the total $\Delta \mathrm{V}$ for the best optimized trajectory was $11.538 \mathrm{~km} / \mathrm{s}$ while the total $\Delta \mathrm{V}$ for the actual trajectory was $9.781 \mathrm{~km} / \mathrm{s}$. This results in a 15.22 percent difference between the two trajectories which leads to a larger difference than the flight times. 


\subsubsection{Cassini Analysis With Perturbations}

When run with perturbations, STOpS was again able to calculate trajectories which maintained similar times of flight for each leg of the trajectory when compared to the actual trajectory utilized by Cassini on its mission. Five cases were run and the average of these trajectories as well as the trajectory with the best cost are analyzed. These optimized trajectories are incidentally also similar to the optimized trajectories without perturbations present. Like the unperturbed trajectories, STOpS was constrained in order to search for only the prograde trajectories with respect to the planet's motion. The perturbations of $J_{2}$, SRP, general relativity as well as all of the planets were included into the optimization. All of the planets were included as the Cassini mission spends large amounts of time both near the inner terresetrial planets as well as the outer gas planets. The optimized trajectory with the best cost can be viewed below in figure 7.7 .

\begin{tabular}{|l|l|l|} 
Sun & Leg 1 \\
Venus & Leg 2 \\
Earth & Leg 3 \\
Jupiter & Leg 4 \\
Saturn & Leg 5 \\
\hline
\end{tabular}




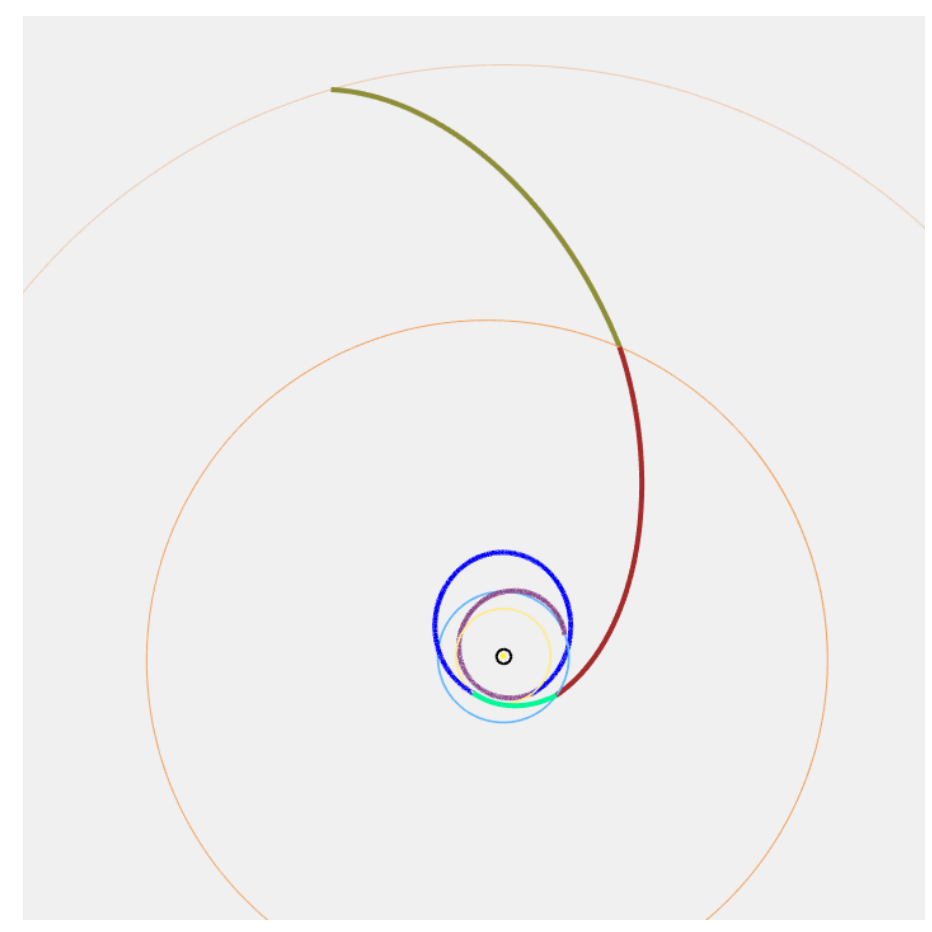

\section{Figure 7.7: Cassini's Optimized Trajectory With Perturbations}

The analysis of the cost parameters for both the perturbed and unperturbed trajectories can be viewed in Table 7.5 in relation to the actual Cassini trajectory. A list $\Delta \mathrm{V}$ expenditures are shown including all $\Delta \mathrm{V}$ values for flybys around each planet. Again, the $\Delta \mathrm{V}$ values corresponding to the flyby portion of the trajectory are only from the change in velocity due to the spacecraft's thruster. The final cost of the trajectories can be viewed as well in comparison to the cost of the actual trajectory. A comparison in flight times between the optimized perturbed and unperturbed trajectories is shown by Table 7.6. These flight times are within reason in comparison to the actual trajectory and are able to create a successful trajectory relative to fuel and time constraints. The total flight time of the best optimized trajectory is 2466 days while the total time of flight for the actual trajectory was 2451 days. This results in just a 0.61 percent difference in flight times between the two trajectories. 


\section{Table 7.5: Cost Parameter Comparison for Cassini's Perturbed Trajectory}

\begin{tabular}{|l|l|l|l|l|}
\hline & $\begin{array}{l}\text { Best } \\
\text { Perturbed }\end{array}$ & $\begin{array}{l}\text { Average } \\
\text { Perturbed }\end{array}$ & $\begin{array}{l}\text { Best } \\
\text { Unperturbed }\end{array}$ & $\begin{array}{l}\text { Actual } \\
\text { Trajectory }\end{array}$ \\
\hline$\Delta$ V Departure $(\mathrm{km} / \mathrm{s})$ & 6.5089 & 5.1519 & 3.9832 & 3.8709 \\
\hline$\Delta$ V Flyby $(\mathrm{km} / \mathrm{s})$ & 0.0497 & 1.4539 & 2.3825 & 0.5489 \\
\hline$\Delta$ V Arrival $(\mathrm{km} / \mathrm{s})$ & 5.3528 & 5.2724 & 5.1718 & 5.3616 \\
\hline Total Cost & 121.5794 & 121.7636 & 117.8762 & 100.265 \\
\hline
\end{tabular}

Table 7.6: Flight Time Comparison for Cassini's Perturbed Trajectory

\begin{tabular}{|l|l|l|l|}
\hline & Best Perturbed & Best Unperturbed & Actual Trajectory \\
\hline Earth & October 111997 & November 19 1997 & October 151997 \\
\hline Venus & May 15 1998 & May 19 1998 & April 26 1998 \\
\hline Venus & June 20 1999 & June 26 1999 & June 24 1999 \\
\hline Earth & August 17 1999 & August 18 1999 & August 18 1999 \\
\hline Jupiter & January 2 2001 & January 11 2001 & December 302000 \\
\hline Saturn & July 12 2004 & September 24 2004 & July 1 2004 \\
\hline
\end{tabular}

There are a few items to consider when comparing the perturbed solution to the unperturbed solution of Cassini's trajectory. The first is that for the perturbed solution, STOpS was able to find a trajectory which did not expend large amounts of fuel during the flyby maneuvers where as this was not the case for the unperturbed solution. The second is that both the best cost as well as the average cost of the trajectory was higher for the perturbed trajectory. The best solution for the perturbed 
trajectory had a cost of 121.5794 and a total $\Delta \mathrm{V}$ value of $11.9114 \mathrm{~km} / \mathrm{s}$ while the best solution for the unperturbed trajectory had a cost of 117.8762 and a total $\Delta \mathrm{V}$ value of $11.5375 \mathrm{~km} / \mathrm{s}$. Finally, for the best optimized trajectory, the perturbed case had flight times which were far more similar to the actual Cassini mission than the times corresponding to the unperturbed trajectory. This is likely due to the fact that the perturbed trajectory is closer to modelling the true dynamics of the system and is therefor more likely to converge on the trajectory obtained by the actual Cassini spacecraft.

\subsection{Voyager 2 Trajectory}

\subsubsection{Voyager 2 Background}

Voyager 1 and 2 are space probes launched in September 51977 and August 20 1977, respectively. They were launched in an effort to visit and obtain data from the outer planets and to eventually model the environment in the outer reaches of the solar system and interstellar space [10]. Given the time that they were launched, the desired destination of all of the planets were already in idealized locations. For this analysis only the Voyager 2 probe will be considered as its trajectory flew past all four of the outer gas giant planets of Jupiter, Saturn, Uranus, and Neptune. A possible trajectory taken by Voyager 2 would not be possible today and will not be possible again until 2150. However ideal the planets positions were at this time, it was still of the utmost importance to determine a trajectory that could reach all of these planets with the smallest amount of $\Delta \mathrm{V}$ and in a time that would sync up with the planets eventual destination at arrival. Such an optimized trajectory was found and Voyager 2 completed its trajectory to become perhaps the most important interplanetary mission of all time. Given that a real optimized trajectory exists that was utilized by Voyager 2, STOpS should be able to develop a trajectory that is very 
similar by optimizing the same set of conditions given on the date of launch.

\subsubsection{Voyager 2 Optimization Setup}

The trajectory panel was setup so that all of the flyby planets and arrival planet of Neptune were specified. The trajectory was given a five year period in order to look for launch dates between 1975 and 1980. The trajectory panel can be viewed in figure 7.8 .

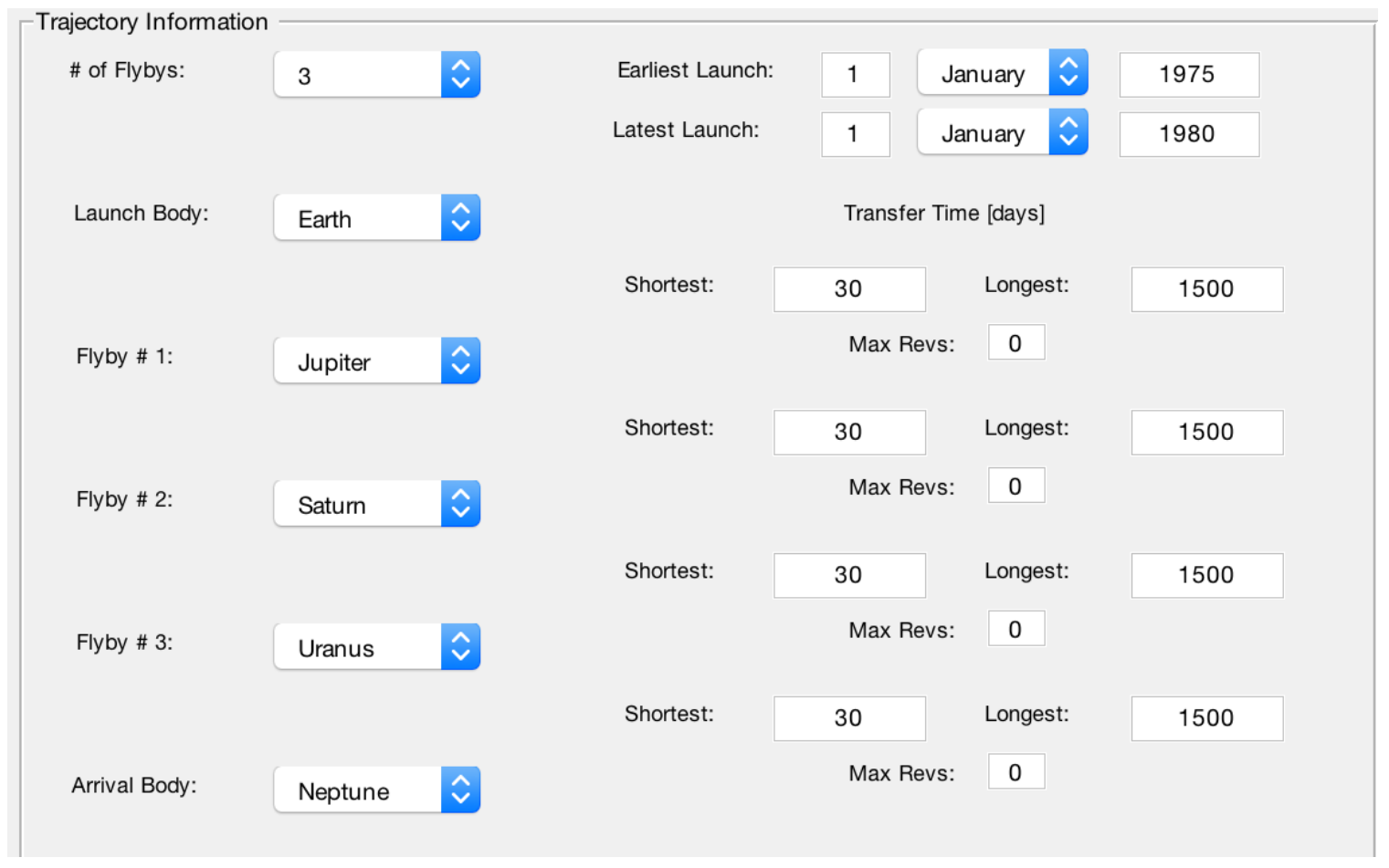

\section{Figure 7.8: Voyager 2 Trajectory Panel}

The cost function which was used for Voyager 2's trajectory, including the weights and parameters chosen can be viewed in figure 7.9. The weights were normalized so that they were the same order of magnitude. Maximum heliocentric energy was chosen to be optimized and the arrival velocity at Neptune was not optimized. This is due to the fact that Voyager 2 did not insert itself into Neptune's orbit and instead continued on a trajectory toward a nearby star. More of an influence was put on the 
$\Delta \mathrm{V}$ weight in order to ensure that the trajectory was feasible based on fuel limitations for the Voyager 2 mission.

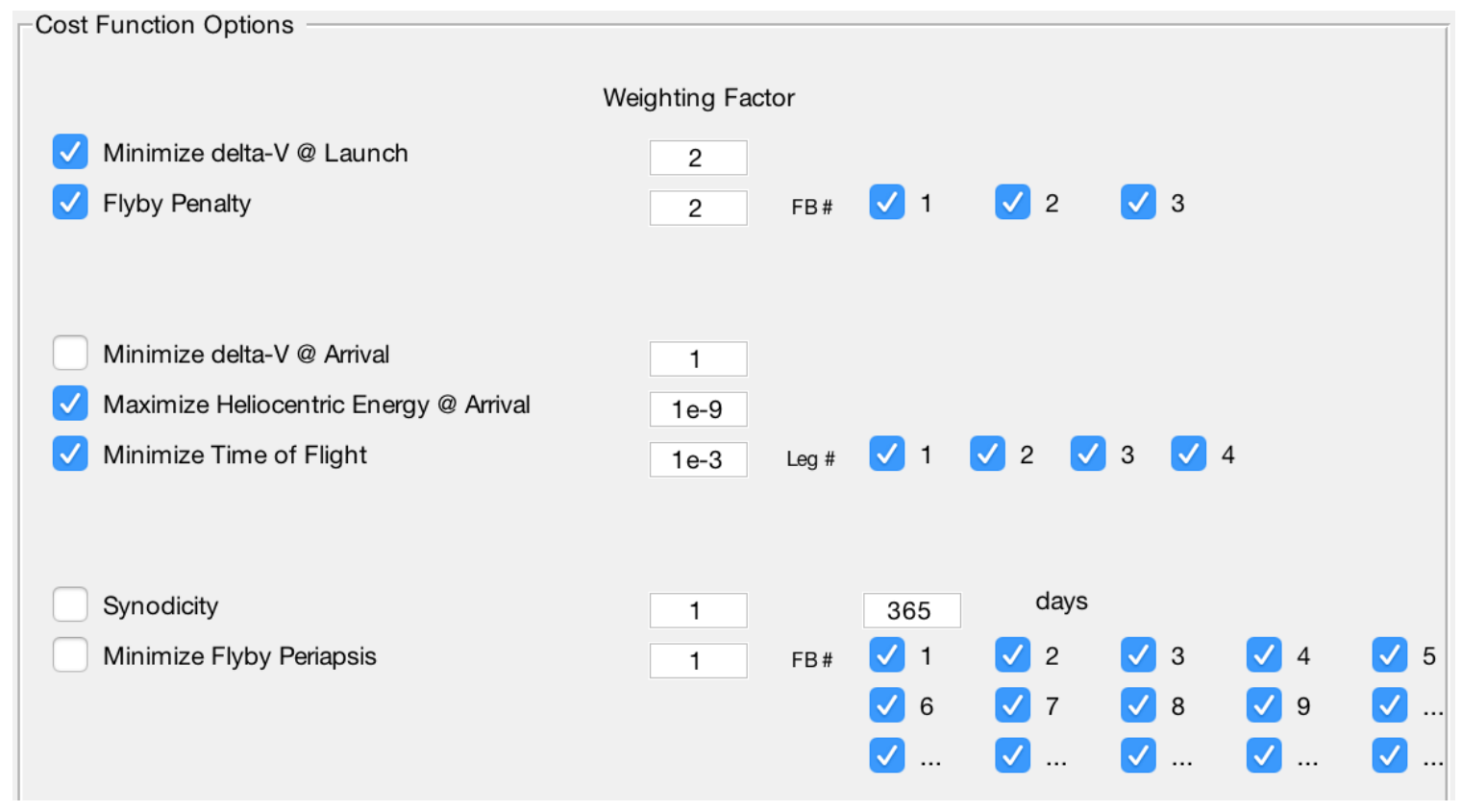

\section{Figure 7.9: Voyager 2 Cost Function Panel}

The algorithms included into this optimization were the genetic algorithm and the differential evolution algorithm. Again, these two algorithms were used because the addition of the other algorithms produced no improvements while slowing the optimization process down. One migration was used and 30 generations were allocated to each algorithm. The optimization options set for the Voyager 2 trajectory are identical to the Cassini trajectory for the same reasons listed under that section. Mainly the population members and number of generations need to be sufficiently high in order for STOpS to arrive at a truly optimal solution. The other parameters listed in this section are base-lined such that the search for solutions does not become random, but also so that there is enough variation in order to prevent stagnation on a non-optimal solution. The optimization panel used for Voyager 2's trajectory can be viewed in figure 7.10 . 


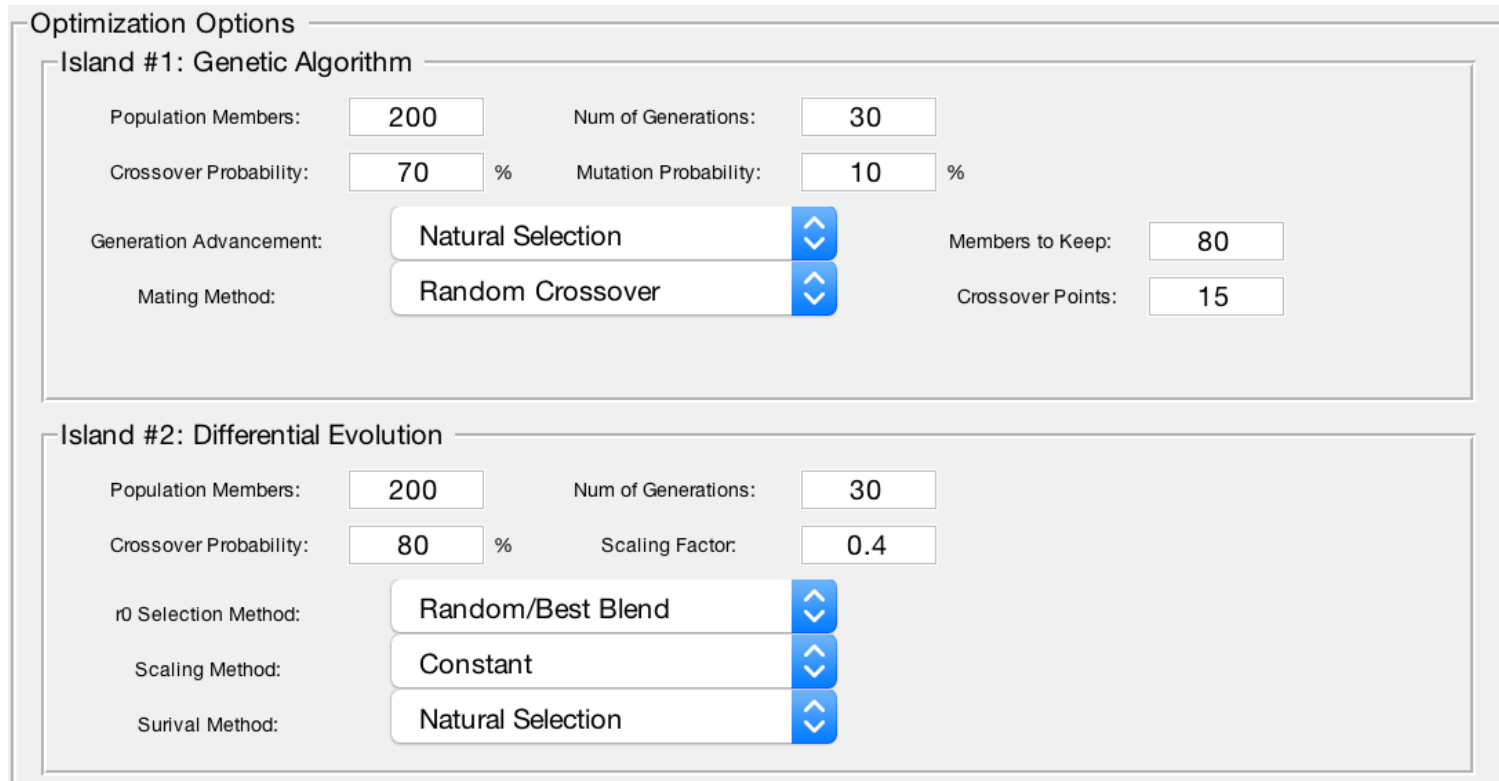

Figure 7.10: Voyager 2's Optimization Panel

\subsubsection{Voyager 2 Analysis Without Perturbations}

When run without perturbations as a two body problem, STOpS was able to obtain a trajectory that resembles the trajectory taken by Voyager 2. Note that depending on the cost function and which parameters are prioritized, the trajectory optimized by STOpS will vary slightly. The optimized trajectory with the best cost can be seen in figure 7.11 in comparison to the actual trajectory taken by Voyager 2 displayed by figure 7.12. The legend includes the planetary orbits as well as each leg of the trajectory.

\begin{tabular}{lll} 
Sun & \multicolumn{1}{|c|}{ Neptune } \\
Earth & $\square$ \\
Leg 1 & Leg 2 \\
Jupiter & Leg 3 \\
Saturn & Leg 4 \\
Uranus & $\square$
\end{tabular}




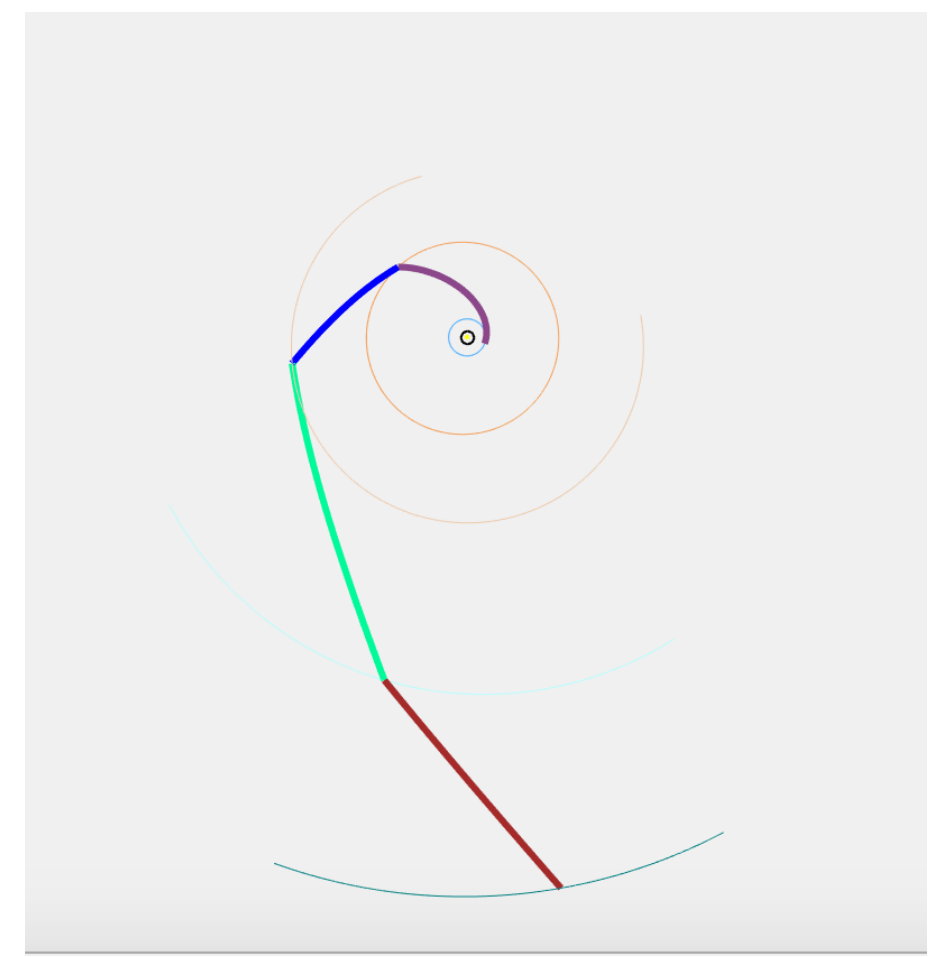

Figure 7.11: Voyager 2's Optimized Trajectory

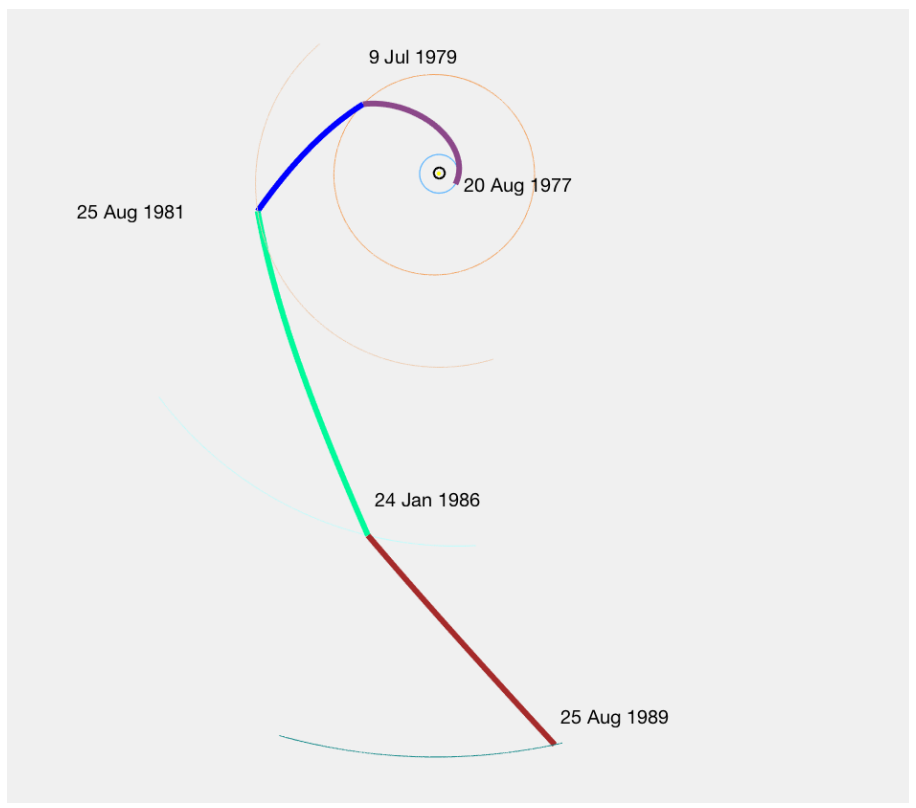

Figure 7.12: Actual Voyager 2 Trajectory

Five optimizations were run for the unperturbed Voyager 2 trajectory. Both the 
average of these trajectories as well as the trajectory with the best cost associated with it are compared with the actual Voyager 2 mission. The cost function parameters for each of these options can be seen in Table 7.7. The total $\Delta \mathrm{V}$ for the best optimized trajectory was $9.6757 \mathrm{~km} / \mathrm{s}$ while the total $\Delta \mathrm{V}$ for the actual trajectory was 10.7431 $\mathrm{km} / \mathrm{s}$. This gives a 9.94 percent difference in $\Delta \mathrm{V}$ values in favor of the optimized trajectory. The overall mission flight time for the best optimized trajectory was very similar to the overall time of flight taken by the actual Voyager 2 mission. When comparing these two trajectories it can be seen that the total time of flight for the best optimized trajectory was 4074 days while the total time of flight for the actual trajectory was 4388. This leads to a 7.16 percent difference in total flight times between the two trajectories. The optimized flight times for each leg of the trajectory can be viewed in Table 7.8 in comparison to the flight times of the actual Voyager 2 mission. These values for both flight times and $\Delta \mathrm{V}$ are within reason for the scope mission since they are within the values performed by the actual Voyager 2 spacecraft.

Table 7.7: Cost Parameters for Voyager 2's Unperturbed Trajectory

\begin{tabular}{|l|l|l|l|}
\hline & $\begin{array}{l}\text { Best } \\
\text { Unperturbed }\end{array}$ & $\begin{array}{l}\text { Average } \\
\text { Unperturbed }\end{array}$ & $\begin{array}{l}\text { Actual } \\
\text { Trajectory }\end{array}$ \\
\hline$\Delta \mathrm{V}$ Departure $(\mathrm{km} / \mathrm{s})$ & 9.6263 & 9.6264 & 10.092 \\
\hline$\Delta \mathrm{V}$ Flyby $(\mathrm{km} / \mathrm{s})$ & 0.0494 & 0.0494 & 0.6511 \\
\hline$\Delta \mathrm{V}$ Arrival $(\mathrm{km} / \mathrm{s})$ & 0 & 0 & 0 \\
\hline Total Cost & 23.4253 & 23.4255 & 25.8541 \\
\hline
\end{tabular}




\section{Table 7.8: Flight Times for Voyager 2's Unperturbed Trajectory}

\begin{tabular}{|c|c|c|}
\hline & Best Unperturbed & Actual Trajectory \\
\hline Earth & September 1 1977 & August 20 1977 \\
\hline Jupiter & May 31 1979 & July 9 1979 \\
\hline Saturn & May 22 1981 & August 25 1981 \\
\hline Uranus & June 301985 & January 21 1986 \\
\hline Neptune & October 27 1988 & August 25 1989 \\
\hline
\end{tabular}

This optimized solution provides a trajectory that can easily intersect Jupiter, Saturn, Uranus, and Neptune with one trip and can therefor be viewed as a reasonable trajectory. The optimized trajectory found by STOpS was able to use a shorter flight time as well use a smaller amount of $\Delta \mathrm{V}$ when compared to the actual Voyager 2 trajectory. Also of note is that there are minimal flyby $\Delta \mathrm{V}$ penalties on all of the gravity assists. This means the optimization was able to find trajectories where the magnitude of the incoming and outgoing velocities on the gravity assists of Jupiter, Saturn, and Uranus were all very similar to each other. This allows the spacecraft to only expend a very small amount of fuel after it leaves Earth's orbit. The gravitational assists are able to provide all of the extra heliocentric energy needed to reach the destination of Neptune for a successful mission.

\subsubsection{Voyager 2 Analysis With Perturbations}

When perturbations are implemented into the optimization process, a very similar trajectory is found compared to the optimized trajectory without perturbations. This trajectory is then incidentally similar to the true trajectory taken by Voyager 2 . The perturbed optimized trajectory with the best cost can be seen in figure 7.13. 

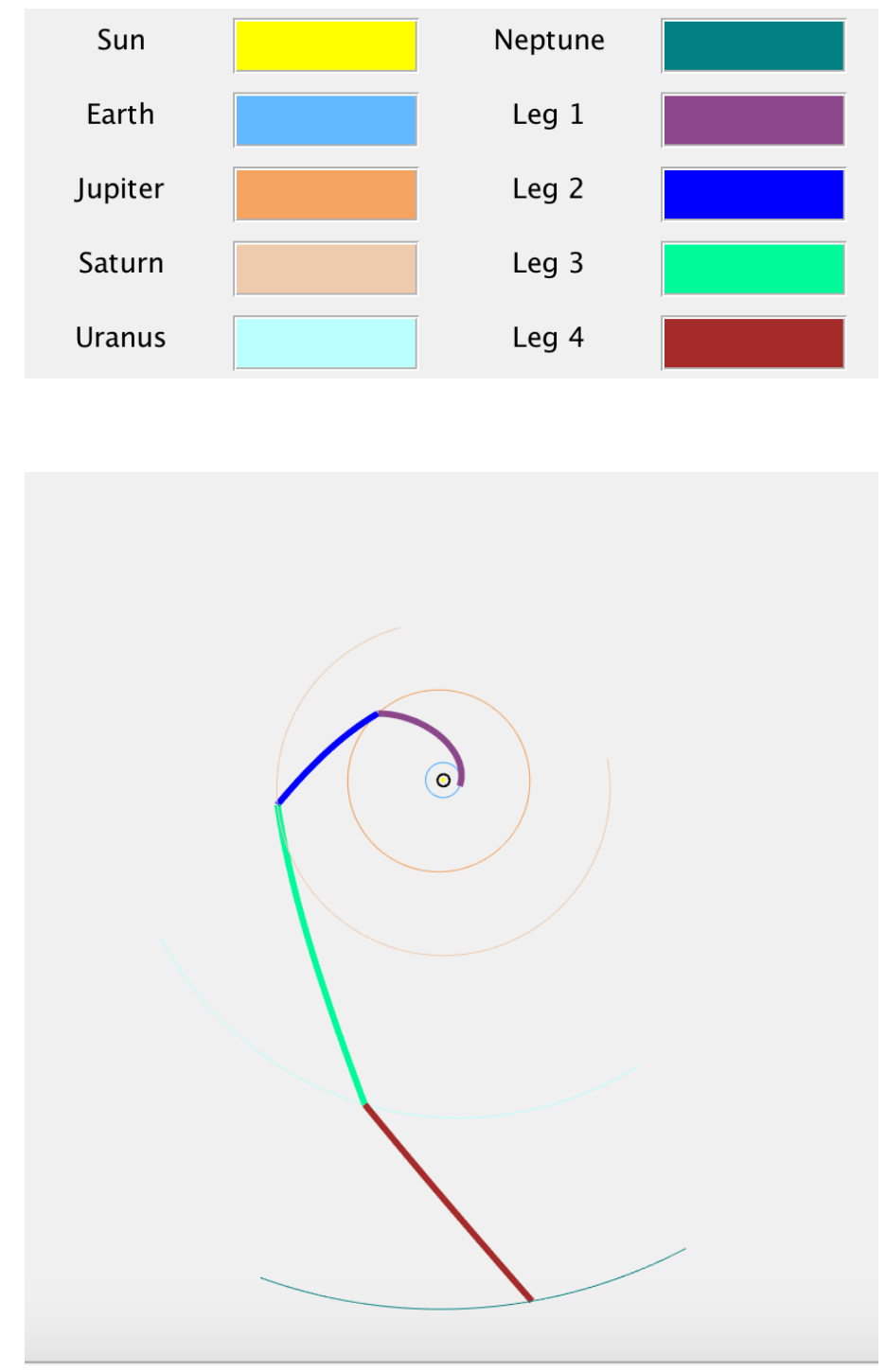

Figure 7.13: Voyager 2's Optimized Trajectory With Perturbations

Again five trials were run and the average of these trajectories as well as the trajectory with the best cost are analyzed. The planetary bodies that were used as perturbations were that of Earth, Jupiter, Saturn, Uranus, and Neptune. Because of the continued acceleration on the spacecraft during its trajectory, the velocity vector leaving Earth has to be modified slightly so that it can correctly arrive at the flyby destination of Jupiter. Although the departure occurs at the same time, the perturbed trajectory has a departure velocity of $[13.4471,36.6775,1.181] \mathrm{km} / \mathrm{s}$ when compared 
to the unperturbed trajectory of $[13.358,36.7124,1.1705] \mathrm{km} / \mathrm{s}$. The cost parameters of the perturbed and unperturbed trajectories are compared with the cost parameters of the actual trajectory as seen in Table 7.9. The departure, flyby, and arrival dates for the perturbed and unperturbed trajectories can be compared to that of the actual Voyager 2 trajectory in Table 7.10 .

Table 7.9: Cost Parameter Comparison for Voyager 2's Perturbed Trajectory

\begin{tabular}{|l|l|l|l|l|}
\hline & $\begin{array}{l}\text { Best } \\
\text { Perturbed }\end{array}$ & $\begin{array}{l}\text { Average } \\
\text { Perturbed }\end{array}$ & $\begin{array}{l}\text { Best } \\
\text { Unperturbed }\end{array}$ & $\begin{array}{l}\text { Actual } \\
\text { Trajectory }\end{array}$ \\
\hline$\Delta \mathrm{V}$ Departure $(\mathrm{km} / \mathrm{s})$ & 9.6259 & 9.9047 & 9.6263 & 10.0920 \\
\hline$\Delta \mathrm{V}$ Flyby $(\mathrm{km} / \mathrm{s})$ & 0.0504 & 0.0267 & 0.0494 & 0.6511 \\
\hline$\Delta \mathrm{V}$ Arrival $(\mathrm{km} / \mathrm{s})$ & 0 & 0 & 0 & 0 \\
\hline Total Cost & 23.4269 & 23.8195 & 23.4253 & 25.8541 \\
\hline
\end{tabular}

Table 7.10: Flight Time Comparison for Voyager 2's Perturbed Trajectory

\begin{tabular}{|l|l|l|l|}
\hline & Best Perturbed & Best Unperturbed & Actual Trajectory \\
\hline Earth & September 1 1997 & September 1 1977 & August 20 1977 \\
\hline Jupiter & May 311979 & May 311979 & July 91979 \\
\hline Saturn & May 22 1981 & May 22 1981 & August 251981 \\
\hline Uranus & June 301985 & June 301985 & January 211986 \\
\hline Neptune & October 281988 & October 271988 & August 251989 \\
\hline
\end{tabular}

The optimized trajectory with perturbations is able to find a trajectory with a 
lower cost than that of the actual trajectory. The average cost of the perturbed trajectory is slightly higher than that of the unperturbed trajectory for the Voyager 2 mission. However both the $\Delta \mathrm{V}$ values as well as the flight times are both very similar for the perturbed and unperturbed cases. When comparing the total $\Delta \mathrm{V}$ values between the perturbed and unperturbed cases we get values of $9.6757 \mathrm{~km} / \mathrm{s}$ and $9.6763 \mathrm{~km} / \mathrm{s}$ respectively. This leads to just a 0.006 percent difference in the $\Delta \mathrm{V}$ values between the two trajectories. This analysis of Voyager 2 has far less variance in the trajectories found than that of the Cassini mission. For the best optimized trajectories, the final arrival at Neptune varies by only one day between the perturbed and unperturbed trajectories. Because the outer planets stay in roughly the same location relative to the Sun throughout the course of the trajectory, STOpS is given fewer opportunities for finding differing trajectories when compared to the Cassini mission. As a result the largest difference that occurs when the perturbations are added is the change in the direction vector of the velocity at departure from Earth. The magnitude of this vector remains the same as can be seen in both $\Delta \mathrm{V}$ values at departure however the direction must change in order to target the correct periapse of Jupiter. Once at Jupiter, both the unperturbed and perturbed trajectory are very similar in their flight times and fuel expenditures. 


\section{Chapter 8}

\section{CONCLUSIONS}

The capability to create orbital optimization programs which utilize evolutionary algorithms has increased over several decades as computers become faster and methods are improved. Several programs such as Copernicus have been created which seek to create the most accurate solution to orbital mechanics problems. As a result there is an ever growing need to improve on these orbital mechanics optimization programs.

It is then desired to develop a program which models the spacecraft environment as accurately as possible so that there can be maximum certainty on the effectiveness of the solution. In order to create such a program the assumption of a two body system with the Sun's gravity as the only acting force on the spacecraft had to be discarded. Instead the dynamics from the perturbations due to multiple environmental effects were added in order to create a more realistic trajectory. However the addition of these dynamics posed problems when it became necessary to implement them into Lambert's problem. In order to accommodate this, a modified Lambert's problem needed to be created in order to handle the dynamics due to environmental perturbations.

This perturbed Lambert's problem was developed which was able to converge on trajectories similar to the two body Lambert's problem. These trajectories were able to be found through the use of Shepperd's method as well as a Runge Kutta Fehlberg 78 integration scheme. These interplanetary trajectories were then coupled with patch conics which included the addition of perturbations during the flyby portions of the trajectory. This produced a model which included environmental perturbations for all sections of the trajectory. 
The perturbed trajectories did vary in both $\Delta \mathrm{V}$ expenditures as well as time of flight. However because the accelerations due to these perturbations are relatively small in comparison to the main gravitational body of the Sun, the perturbed and unperturbed models produce similar results. That is to say that when the best optimized results are compared the $\Delta \mathrm{V}$ differs by $0.3734 \mathrm{~km} / \mathrm{s}$ and $0.0006 \mathrm{~km} / \mathrm{s}$ for the Cassini and Voyager 2 missions respectively. The difference in total flight time between the best optimized perturbed and unperturbed trajectories differed by 35 days and 1 day for Cassini and Voyager 2 respectively. There are larger differences in the perturbed trajectories for the Cassini mission than for the Voyager 2 mission. Because STOpS had more variance in the solutions that were being found for the Cassini trajectory, it should be expected that larger differences in $\Delta \mathrm{V}$ values and flight times will occur. The larger variance comes from the fact that Cassini utilized many flybys around the inner planets which have a shorter orbital period, thus giving many more opportunities for flybys at different locations. When compared to the optimization of Voyager 2, the outer planets stay in roughly the same location relative to the Sun throughout the course of the trajectory, giving STOpS fewer opportunities for finding differing trajectories. Based on the proximity of the perturbed solution to the unperturbed solution, the optimization process present within this work was able to successfully calculate reasonable trajectories for both the mission of Voyager 2 and Cassini. When comparing the perturbed and unperturbed trajectories there is no significant divergence between the two solutions that were found for each trajectory.

It should be noted that the results of the optimized trajectory will change based on both the cost function as well as the optimization algorithms chosen. In order to produce reliable and repeatable trajectories, a large population of trajectories and a large number of generations should be used for the algorithms. Additionally the cost function should be weighted properly so that proper importance is given to each parameter in the cost function. When these specifications are met, the optimized 
trajectories were able to arrive at very similar results when compared to the actual Cassini and Voyager 2 trajectories.

\subsection{Future Work}

While this updated version of STOpS more closely resembles the spacecraft environment than the previous version, there are still many assumptions made within the optimization process. Among the most important of these assumptions is the constraint that the spacecraft cannot perform $\Delta \mathrm{V}$ maneuvers while in transit between planetary bodies. For missions like Cassini where a correction was made between flybys, this assumption can lead to results that are not as optimized as possible given the mission capabilities. Future work should be done in this area to produce a change in velocity at any point in the interplanetary trajectory.

Another large assumption inherent within STOpS is the necessity to specify the planetary flyby bodies before the optimization process begins. A more robust optimization process may be developed in the future which could input solely the departure and arrival planets, and STOpS would be able to search for the planets which would give the best gravitational assists. Also included in this section would be the option to perform flybys around moons within a specific planet system as many of the larger moons can provide large gravitational accelerations at close proximity.

Because of the nature of the perturbed Lambert's solution developed in this work, a large computational time must be devoted in order to optimize these trajectories. Because of the many iterations needed as well as the numerical propagation of trajectories, this can lead to large calculation times when thousands of trajectories are being evaluated at a time. Future work should be done in this area in order to improve the optimization time. This may involve modifying the methodology behind the perturbed Lambert's problem in order to produce a more computationally effi- 
cient solution. Further improvement could be made by transferring the optimization to a different programming language other than Matlab.

One final addition to this work that was not pursued due to time constraints was incorporating orbital synodic periods in order to improve the optimization process. Because the optimization of these trajectories is heavily influenced by the position of the planets for gravitational assists, then multiple optimized trajectories can be found given enough time as the planetary positions will eventually repeat themselves. Voyager 2 as an example had the outer planets in perfect positions during the 1970's in order to reach a trajectory toward Neptune. This specific planetary alignment will not occur again until 2150. It could be useful in the future for STOpS to determine the periodicity of the legs of the trajectories so that similarly optimized trajectories could be found more easily. 


\section{BIBLIOGRAPHY}

[1] A. Cayley. Sur quelques proprits des dterminants gauches. fr die reine und angewandte Mathematik, 32:119-123, 1846.

[2] M. Clerc. Particle Swarm Optimization. London: ISTE, 2006.

[3] H. Curtis. Orbital Mechanics for Engineering Students. 2nd ed. Amsterdam: Elsevier, Butterworth-Heinemann, 2010.

[4] M. Dorigo and T. S. tzl. Ant Colony Optimization. Cambridge, MA: MIT, 2004.

[5] D. Eagle. https://www.mathworks.com/matlabcentral/fileexchange/39703cowell-s-method-for-earth-satellites rkf78, January 2013.

[6] T. J. Fitzgerald. Spacecraft trajectory optimization suite (stops): Optimization of multiple gravity assist spacecraft trajectories using modern optimization techniques. Master's thesis, California Polytechnic State University, 2015.

[7] D. E. Goldberg. Genetic Algorithms in Search, Optimization, and Machine Learning. Reading, MA: Addison-Wesley, 1989.

[8] R. L. Haupt and S. E. Haupt. Practical Genetic Algorithms. 2nd ed. Hoboken, N.J: Wiley-Interscience, 2004.

[9] G. . S. M. Johnson. Copernicus, a generalized trajectory design and optimization system. Technical report, Department of Aerospace Engineering and Engineering Mechanics The University of Texas at Austin, December 2003.

[10] P. A. Kohlhase, C. E. Penzo. Voyager mission description. Space Science 
Reviews, 21:77-101, 1977.

[11] P. M. Morse and H. Feshbach. In Methods of Theoretical Physics, Part I. New York: McGraw-Hill, 1953.

[12] R. M. S. Price, Kenneth V. and J. A. Lampinen. Differential Evolution: A Practical Approach to Global Optimization. Berlin: Springer, 2005.

[13] J. Sauer, Carl G. Midas - mission design and analysis software for the optimization of ballistic interplanetary trajectories. Journal of the Astronautical Sciences, 37:251-259, 1989.

[14] S. W. Shepperd. Universal keplerian state transition matrix. Celestial Mechanics, 35:129-144, February 1985.

[15] Y. H. Troy Goodson, Donald Gray and F. Peralta. Cassini maneuver experience - launch and early cruise. In Guidance, Navigation, and Control Conference and Exhibit, 1998.

[16] S. Urban and P. K. Seidelmann. Explanatory supplement to the Astronomical almanac. Mill Valley, Calif. : University Science Books, 2013.

[17] D. A. Vallado and W. D. McClain. Fundamentals of Astrodynamics and Applications. Dordrecht: Kluwer Academic, 2001.

[18] H. P. J. I. Vega, Francisco Fernandez De and J. Lanchares. The Generalized Island Model. Heidelberg: Springer-Verlag, 2012.

[19] J. Williams. Overview and software architecture of the copernicus trajectory design and optimization system. Elizabeth C.4th International Conference on Astrodynamics Tools and Techniques Madrid; Spain, May 2010. 


\title{
APPENDICES
}

\author{
Appendix A \\ STOPS USER GUIDE
}

STOpS is organized into a graphical user interface so that both inputs into the optimization process as well as analysis and evaluation are user friendly. There are many different panels where the user can change parameters of the optimization process which include the desired trajectory as well as all of the optimization information necessary. For a more detailed look at the STOpS user guide, the reader is encouraged to view Timothy J. Fitzgeralds's thesis [6].

\section{A.1 Optimization Panels}

Located in the top left corner of the STOpS graphical user interface, is a drop down panel which controls all of the important parameters of the optimization process that the user can input. A list of these options and the parameters within them is detailed in this section.

\section{A.1.1 Island Topology}

In this tab it is possible to control which types of evolutionary algorithms to utilize and how they communicate with one another. A maximum of 18 islands can be used with six different types of algorithms. These algorithms can then be connected in

different ways under the different types of topologies available. These algorithms will be able to share information with each other depending on how they are connected within the topology. Also available under this panel are the number of migrations that 
the optimization process will perform. The number of migrations will specify how many times the different algorithms linked within the topology can share solutions with one another.

\section{A.1.2 Trajectory Information}

Trajectory Information is the default panel in the graphical user interface. Under this panel the user can input the arrival, flyby, and arrival planets desired for the trajectory. Also included here are an upper and lower bound on transfer times for each leg of the trajectory. If the user wants a mission from Earth to Mars in 150 days or less then this bound can be specified within the interface and STOpS will not search for any trajectories that take longer than this time.

\section{A.1.3 Optimization Options}

On the next panel it is possible to change parameters within the optimization process of STOpS. Here it is possible to set population size, number of generations, and rate of mutation. The user should change these at the discretion of the mission that they wish to optimize.

\section{A.1.4 Cost Function Options}

Under the next panel are the options to modify the cost function. Here all weights can be adjusted and any parameters in the cost function can be chosen to be included or disregarded. What is specified here will heavily influence the type of trajectory that STOpS will search for. 


\section{A.1.5 Actual Mission}

The actual mission tab allows the user to view the true trajectories of a few interplanetary missions which can be compared with optimized results. These missions include Voyager 1, Voyager 2, Mariner 10, and Curiosity.

\section{A.2 Results of Analysis}

To the direct right of the main page is the analysis of the optimized trajectory. While running, STOpS will display both the current island which is being evaluated as well as which generation of the population is currently being optimized. Once done with the evaluation, STOpS will plot the trajectory in three dimensional coordinates. This plot can be rotated or zoomed in to show different angles and aspects of the trajectory.

There is a tab on this evaluation section that will also show all of the important optimized parameters of the code. A cost analysis shows the costs associated with the highest lowest and average population member for every generation. This is useful for the user to see that the optimization process is in fact finding lower cost trajectories with successive generations. It also helps to modify the number of generations needed if the user would like a more optimized cost or if the cost has leveled off in earlier generations.

The next section of the analysis shows the important parameters of the optimized trajectory. These parameters include final transfer times from planet to planet, $\Delta \mathrm{V}$ needed in order to achieve these trajectories by the spacecraft, $\Delta \mathrm{V}$ provided by the planets during gravity assist maneuvers, heliocentric energy at the end of the trajectory, and finally the cost associated with the final optimized trajectory. 